\title{
The Tomato Kinase Pti1 Contributes to Production of Reactive Oxygen Species in Response to Two Flagellin-Derived Peptides and Promotes Resistance to Pseudomonas syringae Infection
}

\author{
Simon Schwizer, ${ }^{1,2}$ Christine M. Kraus, ${ }^{1,2}$ Diane M. Dunham, ${ }^{1}$ Yi Zheng, ${ }^{1}$ Noé Fernandez-Pozo, ${ }^{1}$ \\ Marina A. Pombo, ${ }^{1}$ Zhangjun Fei, ${ }^{1,2}$ Suma Chakravarthy, ${ }^{2}$ and Gregory B. Martin ${ }^{1,2,+}$ \\ ${ }^{1}$ Boyce Thompson Institute for Plant Research, Ithaca, NY 14853, U.S.A.; and ${ }^{2}$ Plant Pathology and Plant-Microbe Biology \\ Section, School of Integrative Plant Science, Cornell University, Ithaca, NY 14853, U.S.A.
}

Accepted 22 May 2017.

\begin{abstract}
The Pti1 kinase was identified from a reverse genetic screen as contributing to pattern-triggered immunity (PTI) against Pseudomonas syringae pv. tomato (Pst). The tomato genome has two Ptil genes, referred to as Ptila and Ptilb. A hairpin-Pti1 (hpPti1) construct was developed and was used to generate two independent stable transgenic tomato lines that had reduced transcript abundance of both genes. In response to $P$. syringae pv. tomato inoculation, these hpPti1 plants developed more severe disease symptoms, supported higher bacterial populations, and had reduced transcript accumulation of PTI-associated genes, as compared with wild-type plants. In response to two flagellin-derived peptides, the hpPti1 plants produced lesser amounts of reactive oxygen species (ROS) but showed no difference in mitogen-activated protein kinase (MAPK). Synthetic Ptila and Ptilb genes designed to avoid silencing were transiently expressed in the hpPti1 plants and restored the ability of the plants to produce wild-type levels of ROS. Our results identify a new component of PTI in tomato that, because it affects ROS production but not MAPK signaling, appears to act early in the immune response.
\end{abstract}

Current address for Simon Schwizer: Department of Plant Pathology and Microbiology, University of California, Riverside, Riverside, CA 92521, U.S.A.

Current address for Marina A. Pombo: Instituto de Fisiología Vegetal, La Plata 1900, Argentina.

Current address for Suma Chakravarthy: Animal Plant and Health Inspection Service-Biotechnology Regulatory Services, United States Department of Agriculture, River Road, Riverdale, MD 20737, U.S.A.

Gene sequences are available from the Sol Genomics Network database under the following accession numbers: SlPtila (Solyc12g098980), SlPtilb (Solyc05g053230), NbPtila (Niben101Scf01236g02003), NbPtilb (Niben101Scf01334g04008), NbPtilc (Niben101Scf01671g04002), and NbPtild (Niben101Scf01820g00026). RNA-Seq reads have been deposited in the National Center for Biotechnology Information Sequence Read Archive under accession number SRP076863 and analyzed data is available from the Tomato Functional Genomics database under accession number D014.

${ }^{\dagger}$ Corresponding author: Gregory B. Martin; Email: gbm7@cornell.edu

*The $e$-Xtra logo stands for "electronic extra" and indicates that six supplementary figures, four supplementary tables, and three supplementary datasets are published online

(C) 2017 The American Phytopathological Society
The interaction of tomato (Solanum lycopersicum) with Pseudomonas syringae pv. tomato both causes a persistent disease of tomatoes and also provides a powerful model system for understanding bacterial pathogenesis and the plant immune system (Jones 1991; Pedley and Martin 2003; Young et al. 1986). In common with other plants, tomato resists attack by microbial pathogens by employing a sophisticated, two-layered immune system (Cook et al. 2015; Dodds and Rathjen 2010; Jones and Dangl 2006). The first layer of defense involves pattern recognition receptors (PRRs) located at the plasma membrane that are able to perceive extracellular microbe-associated molecular patterns (MAMPs) (Boller and Felix 2009; Couto and Zipfel 2016; Hohmann et al. 2017; Zipfel 2014). These MAMPs are typically conserved components present in essential pathogen structures, such as the flagellin protein in the bacterial flagellum, which contains two MAMPs, flg22 and flgII-28 (Bent and Mackey 2007; Cai et al. 2011; Clarke et al. 2013; Felix et al. 1999).

One of the best-characterized PRRs is FLS2, which detects flg22 and occurs in Arabidopsis, tomato, and other plants (Boller and Felix 2009; Chinchilla et al. 2006; Gómez-Gómez and Boller 2000; Robatzek et al. 2007). FLS2, upon binding flg22, initiates a signaling cascade, leading to a variety of responses, including production of reactive oxygen species (ROS), activation of mitogenactivated protein kinase (MAPK) cascades, and induction of defense-related genes (Zipfel 2014). These responses along with production of antimicrobial compounds and cell-wall reinforcements halt pathogen invasion and are collectively referred to as pattern-triggered immunity (PTI) (Boller and Felix 2009). The flgII-28 peptide is recognized by tomato, potato, and pepper, and the gene encoding the cognate PRR, referred to as FLS3, was recently identified using a mapping-by-sequencing approach (Clarke et al. 2013; Hind et al. 2016).

To overcome these defense responses and cause disease, pathogenic microbes have evolved virulence proteins (effectors) that are typically translocated into the plant cell to interfere with pathogen detection or interrupt PTI signaling (Dou and Zhou 2012; Macho and Zipfel 2015). P. syringae pv. tomato, like many other bacterial pathogens, employs a type III secretion system, which acts as a molecular syringe to enable injection of approximately 30 effector proteins into the plant cell (Block and Alfano 2011; Buell et al. 2003; Cunnac et al. 2011; Lindeberg et al. 2012). Two of these, AvrPto and AvrPtoB, act early during the PTI response, to interfere with the FLS2 and FLS3 receptor complexes to prevent an effective induction of PTI (Cheng et al. 2011; He et al. 
2006; Hind et al. 2016; Kvitko et al. 2009; Martin 2012; Shan et al. 2008; Xiang et al. 2008).

To defend themselves against the detrimental consequences of effectors, plants have evolved a second layer of defense involving resistance $(\mathrm{R})$ proteins, which are capable of detecting the presence or action of effector proteins (Dodds and Rathjen 2010; Jones and Dangl 2006). In tomato, the Pto protein kinase forms a complex with the nucleotide-binding leucine-rich repeat (NB-LRR) protein Prf and binds AvrPto or AvrPtoB (Dong et al. 2009; Martin et al. 1993; Mucyn et al. 2006; Salmeron et al. 1996; Xing et al. 2007). This interaction induces a strong defense response, referred to as effector-triggered immunity (ETI), resulting in programmed cell death (PCD) of the infected tissue and inhibition of pathogen growth (Jones and Dangl 2006; Pedley and Martin 2003). It has been proposed that Pto evolved as a 'decoy' to mimic the kinase domains of PRRs, such as FLS2 and FLS3, that are targeted by AvrPto and AvrPtoB (Martin 2012; van der Hoorn and Kamoun 2008; Xiang et al. 2008; Xing et al. 2007; Zhou and Chai 2008).

The Ptil serine-threonine kinase was originally identified from a yeast two-hybrid screen as a Pto interactor and was implicated in ETI (Zhou et al. 1995). Overexpression of tomato Ptil in a stable transgenic tobacco line resulted in enhanced cell death in response to $P$. syringae pv. tabaci carrying avrPto, and it was concluded that Ptil amplifies the Pto signaling response (Zhou et al. 1995). A possible role for Ptil in ETI was supported by the observation that Pto specifically phosphorylates Ptil but Ptil does not phosphorylate Pto, suggesting that Ptil functions directly downstream of Pto (Sessa et al. 1998, 2000; Zhou et al. 1995). However, subsequent mutational analysis of Pto revealed several substitution mutants that are unable to phosphorylate Pti1 but still trigger Prf-mediated cell death when transiently overexpressed in Nicotiana benthamiana (Wu et al. 2004). Importantly, a stable transgenic tomato line overexpressing $P t{ }^{G 50 S}$, a variant that lacks kinase activity and does not interact with Ptil in yeast, confers resistance to $P$. syringae pv. tomato strains expressing avrPto (Mathieu et al. 2014; Xiao et al. 2003). Later work found that the response to AvrPto in tobacco differs from tomato and depends on an unidentified resistance protein (Nguyen et al. 2010a; Shan et al. 2000; Yeam et al. 2010). Collectively, these observations suggest that Ptil does not play a role in ETI.

Here, we describe the discovery that Ptil appears to act early in the PTI response by inducing ROS production in response to flagellin perception, influencing the expression of defenserelated genes and enhancing resistance to $P$. syringae pv. tomato.

\section{RESULTS}

\section{Cell death suppression assay identifies Ptil as contributing to PTI.}

Protein kinases are known to play various roles in PTI (Couto and Zipfel 2016; Yamaguchi et al. 2013; Zipfel and Oldroyd 2017). Therefore, to identify new components of PTI, we cloned DNA sequences from 129 receptor-like cytoplasmic kinases (RLCKs) and other protein kinase-encoding genes from tomato and used these for virus-induced gene silencing (VIGS) in N. benthamiana. Each gene-silenced plant was then examined for altered defense responses, using an assay in which PTI is induced by a nonpathogenic bacterial strain, followed by overlap-infiltration of a pathogenic strain (Chakravarthy et al. 2010). If the silenced gene plays a role in PTI, then immunity is not fully induced by the nonpathogenic strain and disease-associated cell death occurs more rapidly in the overlapping area. The Ptil gene was identified in this screen, which was unexpected, because it had previously been identified as playing a role in ETI (Zhou et al. 1995).

Inspection of the tomato genome sequence revealed that there are two Ptil genes, which we refer to as SlPtila (Solyc12g098980) and SlPtilb (Solyc05g053230) (Supplementary Table S1). The genes are $88 \%$ identical at the nucleotide level and their predicted proteins are $93 \%$ identical (Supplementary Fig. S1). Ptila encodes the protein originally identified as interacting with Pto (Zhou et al. 1995). From RNA sequencing (RNA-Seq) data generated previously (Rosli et al. 2013), we determined that Ptila is expressed in tomato leaves, though its expression is not specifically affected by inducers of PTI (it is induced in mock-inoculations after $6 \mathrm{~h}$, as compared with the initial 30 min time point, suggesting possible stress responsiveness [Supplementary Fig. S2]). Ptilb is expressed at a basal level in leaves and its transcript abundance increased significantly in response to the MAMPs csp22 (a 22-amino acid peptide from a bacterial cold-shock protein) and flgII-28 as well as $P$. fluorescens (having both $\mathrm{flg} 22$ and flgII-28) but not in response to Agrobacterium tumefaciens, which has a flagellin that does not trigger transcriptional change in tomato (Rosli et al. 2013). Ptilb transcript abundance also increased in response to inoculation with $P$. syringae pv. tomato and was reduced in the presence of the effectors AvrPto and AvrPtoB.

$N$. benthamiana is an allotetraploid and, as expected, we identified four Ptil genes in the genome sequence of this species, which we refer to as NbPtila (Niben101Scf01236g02003), NbPtilb (Niben101Scf01334g04008), NbPtilc (Niben101Scf01671g04002), and NbPtild (Niben101Scf01820g00026) (Bombarely et al. 2012) (Fig. 1A). To further characterize Ptil in N. benthamiana, we used these gene sequences and the Sol Genomics Network (SGN) VIGS tool (Fernandez-Pozo et al. 2015a) to design two constructs, designated $\mathrm{NbP} 1$ and $\mathrm{NbP}$, each of which was expected to silence all four Ptil genes in N. benthamiana (Supplementary Table S2). These two constructs were used for VIGS, along with an NbFLS2 construct (as a positive control) and an Escherichia coli-derived DNA fragment (EC1, as a negative control) (Rosli et al. 2013). The cell death suppression assay was performed by inducing PTI with $P$. fluorescens 55, followed $7 \mathrm{~h}$ later by overlap-infiltration of DC3000 4 hopQ1-1, which causes disease in N. benthamiana (Chakravarthy et al. 2010; Rosli et al. 2013). As expected, silencing of FLS2, which is known to diminish the response to bacterial flagellin, prevented full induction of PTI, thus resulting in increased disease-associated cell death in the overlapping area compared with EC1 control plants, whose PTI response was not impaired (Fig. 1B and C). Importantly, silencing with either NbP1 or $\mathrm{NbP} 2$ caused increased disease in the overlapping area, as compared with the negative control, indicating a compromised PTI response, although the disease was less than that observed in FLS2-silenced plants (Fig. 1B and C). To evaluate the silencing efficiency of the two VIGS constructs, we performed quantitative real time polymerase chain reaction (qPCR) on NbP1- and NbP2-silenced plants and found that Ptil transcript abundance was reduced to less than $20 \%$ of the level in the EC1 control plants (Fig. 1D). Although our Ptil primers were expected to amplify transcripts of the $N$. benthamiana Ptila, Ptilb, and Ptilc genes, sequencing of the qPCR products from the EC1 control plants revealed only transcripts derived from NbPtilb and NbPtilc. Using previously generated RNA-Seq data (Pombo et al. 2014), we examined reads per kilobase of transcript per million mapped reads (RPKM) and discovered that both $N b P t i l b$ and NbPtilc are highly expressed in N. benthamiana leaves, whereas NbPtila and NbPtild have a much lower transcript abundance (Fig. 1A). Based on the VIGS experiments and these expression levels, we conclude that, among the Ptil genes in N. benthamiana, one or both NbPtilb or NbPtilc are the main contributors to PTI, and the robust silencing of these genes likely explains the observed impact on cell death suppression in the PTI assay.

\section{Transgenic tomato plants silenced for Ptila and Ptilb are more susceptible to $P$. syringae pv. tomato infection.}

To test whether the Ptil genes contribute to PTI in tomato, we developed a hairpin RNA interference (RNAi) construct 
designed to silence the two Ptil genes in this species (Fig. 2A). The construct was used to develop two independent stable transgenic lines in the Rio Grande-prf3 background (RG-prf3, lacking a functional Prf) (Salmeron et al. 1996). One line (F2736) has a single-copy, homozygous, hpPti1 integration and the other line (F10-10) carries multiple copies of the hpPtil construct. For each of these two lines, we identified an 'azygous' control line that was derived from the original transformation event but which had lost the one or more of the transgenes in subsequent generations, due to segregation. To determine the degree of Ptil silencing and to characterize the transcriptome of these plants, we performed RNA-Seq on F27-36 and F10-10 hpPti1 plants (and on one of the azygous controls) that had been

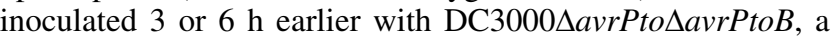
$P$. syringae pv. tomato strain that has reduced virulence and is used to detect subtle changes in host response (Lin and Martin 2005; Kvitko et al. 2009; Rosli et al. 2013). In the hpPti1 lines, the transcript levels of Ptila and Ptilb were reduced to about 35 and $25 \%$, respectively, of the level in the azygous control line (Fig. 2B; Supplementary Fig. S3A). We also inoculated

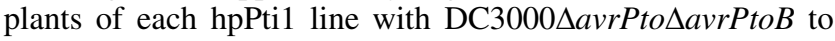
compare their disease symptoms and bacterial populations with azygous and RG-prf3 control plants. Severe disease symptoms were observed on hpPtil plants compared with azygous and RG-prf3 plants, which developed only moderate disease (Fig. 2C). The hpPti1 plants supported about threefold greater bacterial populations compared with the azygous and RG-prf3 controls (Fig. 2D). Together, these observations indicate that, in tomato, one or both of the Ptil kinases contribute to PTI in response to DC3000_avrPto $\Delta a v r P t o B$.

\section{RNA-Seq analysis reveals overlap}

between genes induced by the Ptil proteins and PTI.

To gain insight into the possible roles of Ptila and Ptilb, we further analyzed our RNA-Seq data from the two hpPti1 lines treated with DC3000 $\Delta a v r P t o \Delta a v r P t o B$, to identify genes whose transcript abundance is either reduced or increased in the absence of these kinases. We observed that more genes were affected in the multicopy hpPti1 line (F10-10) than in the single-copy line (F27-36) (Fig. 3A; Supplementary Dataset S1). Because both hpPtil lines showed the same degree of compromised resistance to $P$. syringae pv. tomato, we focused on the subset of genes that were affected in both lines. There were only 26 genes whose transcript abundance was less in both hpPtil lines (i.e., their expression was induced in the presence of Ptila and Ptilb), and only 11 genes whose transcript abundance was greater in both
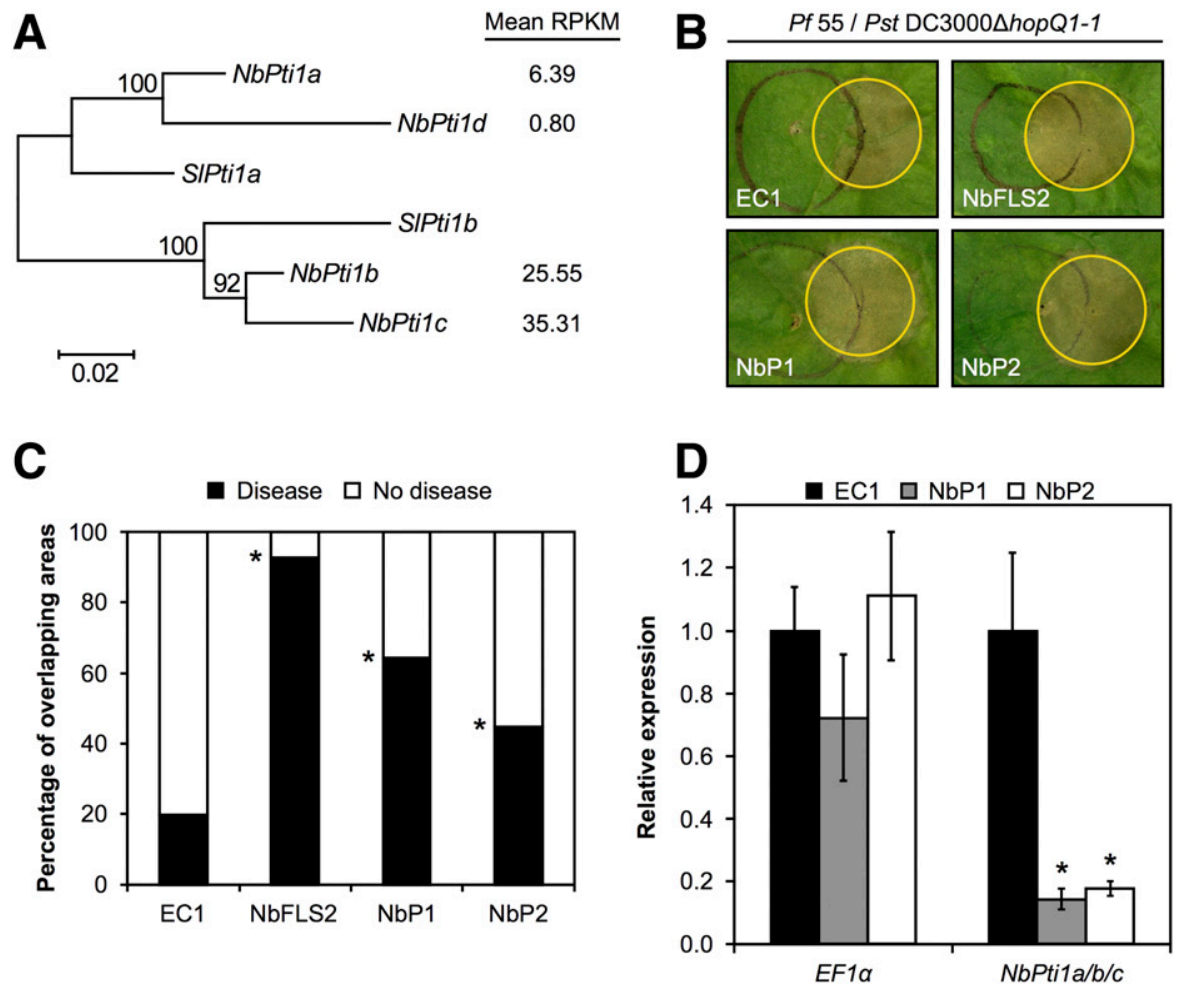

Fig. 1. Silencing Ptil compromises pattern-triggered immunity (PTI) in Nicotiana benthamiana. A, Phylogenetic tree based on nucleotide sequences showing the relationship of the four Ptil genes in N. benthamiana, i.e., NbPtila, NbPtilb, NbPtilc, and NbPtild, to the two Ptil genes in tomato, SlPtila and SlPtilb. Bootstrap percentages are indicated at the branches. Transcript abundance (as mean reads per kilobase of transcript per million mapped reads [RPKM] from three replicates) for each NbPtil gene in mock-treated $N$. benthamiana leaves is shown (Pombo et al. 2014). B, N. benthamiana seedlings were inoculated with Tobacco rattle virus-based virus-induced gene silencing (VIGS) constructs for EC1 (negative control), NbFLS2 (positive control), NbP1, or NbP2 (each construct was designed to silence all four NbPtil genes). Six weeks later, leaves of these plants were syringe-infiltrated with nonpathogenic Pf 55 (black circles) to induce PTI, followed $7 \mathrm{~h}$ later with disease-causing DC3000 $h$ hopQ1-1 (lighter circles) in partially overlapping areas. Compromised induction of PTI by $P f 55$ as a result of gene silencing leads to faster disease development in the overlapping region. Photographs of representative plants were taken 5 days after infiltration. C, Quantification of disease development in the silenced leaves shown in B. Leaf circles with more than $25 \%$ of the overlapping area showing cell death were counted as having disease. For each silencing construct, seven plants were used with two leaves per plant and four circles per leaf, for a total of 56 overlapping circles. Scoring was done 5 days after infiltration. Asterisks indicate significant differences compared with the EC1 control group, using Fisher's exact test. $P$ values are $<0.0001$ for $\mathrm{NbFLS} 2,<0.0001$ for $\mathrm{NbP} 1$, and 0.004 for $\mathrm{NbP} 2$. This experiment was performed twice with similar results. D, Quantitative polymerase chain reaction (PCR) assay to determine the degree of silencing of the NbPtil genes in $N$. benthamiana VIGS plants. The oligonucleotides were designed to detect NbPtila, NbPtilb, and NbPtilc, but sequencing of the PCR products confirmed amplification of transcripts from only NbPtilb and NbPtilc. Transcript levels of $\mathrm{NbPtilb/c}$ in each plant were normalized to PP2A (Liu et al. 2012) and are shown in relation to the EC1 control group. EF1 $\alpha$ is shown as an internal control. Each group contained four or five plants. Graph shows mean $\pm 95 \%$ confidence interval. Asterisks indicate significant differences compared with the control group, based on a Kruskal-Wallis test followed by a Steel-Dwass post hoc test. $P$ values are 0.0376 for NbP1 and 0.0232 for NbP2. 
hpPti1 lines (i.e., their expression was suppressed in the presence of Ptila and Ptilb [Fig. 3A]). Some of the genes with reduced transcript abundance in the hpPtil lines have been implicated in defense responses, including the pathogenesis-related $(P R)-1 b$ gene (Chen et al. 2014) (Solyc00g174340) and the cathepsin L-like cysteine proteinase genes Rcr3 (Solyc02g076980) and Pipl (Solyc02g077040) that were recently reported to play an important role in $P$. syringae pv. tomato resistance in tomato (Ilyas et al. 2015; Richau et al. 2012; Shindo et al. 2016) (Fig. 3B). To more generally investigate whether these differentially expressed genes are associated with PTI, we compared them with our previously published set of genes that are induced or suppressed after treatment with the MAMP flgII-28 (Cai et al. 2011; Rosli et al. 2013). Of the 26 genes with increased transcript abundance when the Ptil kinases were present, 19 were also induced by flgII-28 (73\%). Of the 11 genes with reduced transcript abundance, none were suppressed by flgII-28; there are also five Pti1-suppressed genes induced by flgII-28. Gene Ontology (GO) term analysis of the 26 Ptil-induced and 11 Ptil-suppressed genes showed the former to be predominantly associated with plant defense, whereas the latter are associated with biosynthetic and metabolic processes (Fig. 3C; Supplementary Dataset S2). These analyses are consistent with our disease and bacterial population assays in supporting a role for one or both of the Ptil kinases in the PTI response to $P$. syringae pv. tomato.

\section{Silencing of Ptila and Ptilb negatively impacts ROS production associated with PTI.}

Flagellin-derived peptides (i.e., flg22 and flgII-28) are important MAMPs associated with $P$. syringae pv. tomato-mediated PTI in both tomato and $N$. benthamiana (Chakravarthy et al. 2010; Rosli et al. 2013). To further investigate a role of the Pti1 kinases in PTI, we used two standard assays to test for the host response to these peptides, i.e., activation of MAPK cascades and generation of ROS (Nguyen et al. 2010b). Because we observed no difference in silencing efficiency or disease susceptibility between the single- and the multicopy hpPtil lines, we performed these assays with only the single-copy line (F27-36). To detect MAPK activation, leaf discs were incubated with either flg22, flgII-28, or water, as a control, and phosphorylated MAPKs were detected with an antibody. Although both flg22 and flgII-28 induced MAPK phosphorylation, there was no difference in this response between the hpPti1 and azygous control plants at the lowest concentration of each peptide that reliably activated MAPKs (10 nM for flg22 and $25 \mathrm{nM}$ for flgII-28 [Fig. 4A]). To measure ROS production, we used leaf discs in a
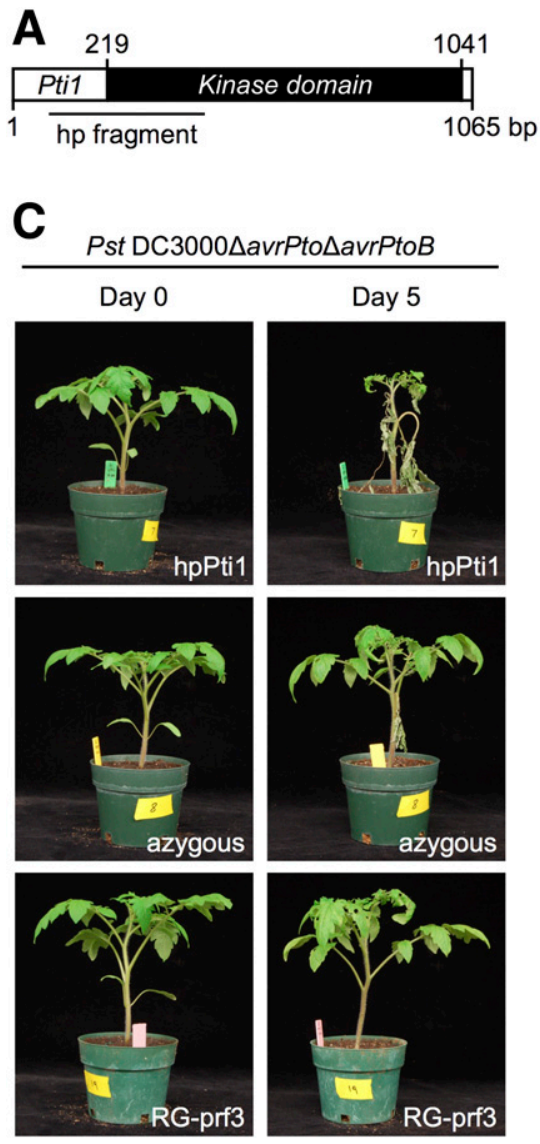

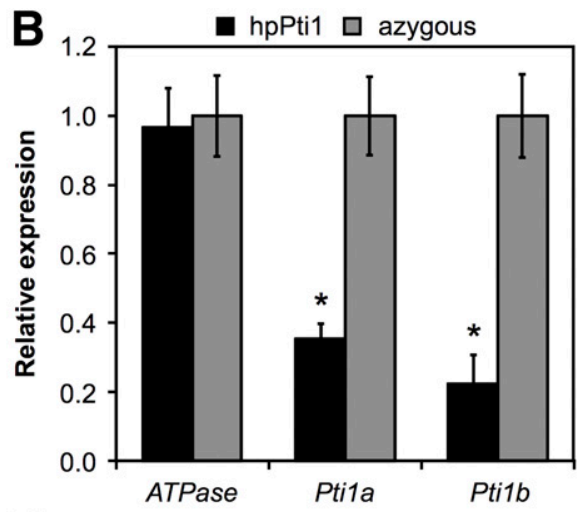

D

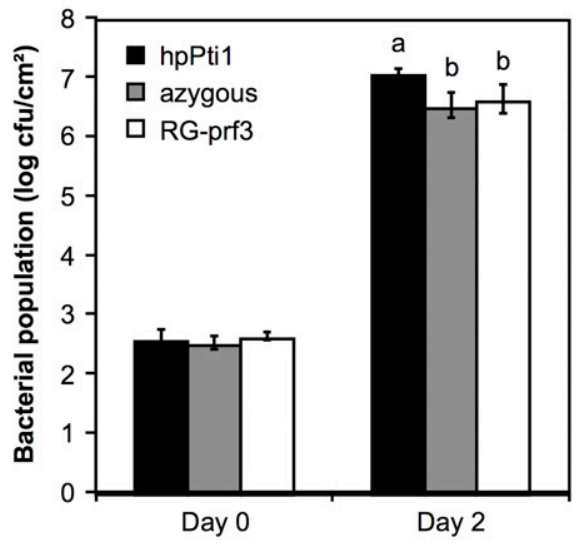

Fig. 2. Transgenic tomato plants silenced for Ptila and Ptilb are more susceptible to infection by DC3000 $\Delta a v r P t o \Delta a v r P t o B$. A, Schematic representation of the tomato Ptila and Ptilb genes, with the kinase domain highlighted in black. The numbers indicate nucleotide positions of the $5^{\prime}$ and $3^{\prime}$ ends and the location of the kinase domain. The origin of the hairpin (hp) fragment is shown below the gene. B, Relative transcript abundance of Ptila and Ptilb in homozygous single-copy hairpin-Pti1 (hpPti1) plants (F27-36) compared with azygous control plants. ATPase is shown as a control. Transcript levels are based on RNA-Seq reads from the 6-h dataset of three plants per genotype normalized to CBL1 (Pombo et al. 2014). Bars show mean $\pm 99 \%$ confidence interval. Asterisks indicate significant differences between hpPtil and azygous plants. $P$ values are $<0.0001$ for both Ptila and Ptilb (based on a false discovery rate correction). C, Fourweek-old transgenic hpPti1 plants (F27-36) along with azygous control plants and progenitor RG-prf3 plants were vacuum-infiltrated with $5 \times 10^{4} \mathrm{CFU}$ of DC3000 avrPto $\Delta a v r$ PtoB per milliliter and disease symptoms were monitored. Photographs of the same representative plants are shown before infiltration (Day 0) and 5 days after infiltration (Day 5). Seven plants for each genotype were tested. D, Bacterial populations were determined in the plants shown in C. Samples were taken after infiltration (Day 0) and 2 days later (Day 2). Bars show the mean $\pm 99 \%$ confidence interval. Different letters indicate significant differences based on a one-way analysis of variance followed by Tukey's honest significant difference post hoc test. $P$ values are 0.0002 for hpPti1 versus azygous and 0.0019 for hpPti1 versus RG-prf3. This experiment was performed four times with similar results. 
chemiluminescence-based assay (Chakravarthy et al. 2010; Clarke et al. 2013). We observed a reduction in ROS production to approximately $50 \%$ in hpPtil plants, as compared with azygous control plants, despite using relatively high concentrations of flg22 and flgII-28 (100 nM each [Fig. 4B]). These observations suggest that one or both of the tomato Ptil kinases function in a signaling pathway activated by flagellin perception upstream of ROS production but independent of the MAPKs monitored by this assay.

\section{Transient complementation demonstrates that either Ptila or Ptilb can restore ROS production to the hpPtil plants.}

In addition to the one or more intended genes, RNAi can silence other nontarget genes, possibly leading to misinterpretations (Senthil-Kumar and Mysore 2011). To determine if the reduced ROS production in response to flg22 and flgII-28 is indeed due to silencing of the Ptil genes, we developed synthetic Ptila and Ptilb genes that would not be silenced by the hpPtil construct (Kumar et al. 2006; Wu et al. 2016). Specifically, the 5' portion of each gene was altered so that it did not have a contiguous region of more than 18 nucleotides identical to the hpPtil fragment that could trigger RNA silencing. The synthetic regions encoded identical amino acid sequences as the wild-type proteins and were fused to the $3^{\prime}$ wild-type sequence of the respective gene (Fig. 5A; Supplementary Fig. S4). To verify that these synthetic Ptil variants, referred to as synPtila and synPtilb, are expressed and evade silencing, we transiently expressed synPtila and synPtilb or wild-type Ptila and Ptilb in N. benthamiana leaves in the presence of the hpPtil construct (Fig. 5B). Accumulation of the synthetic proteins was not significantly different from that of the wild-type proteins when coexpressed with the unrelated hpBti9 construct (Zeng et al. 2011). However, when coexpressed with the hpPtil construct, accumulation of the wild-type Ptil proteins was strongly diminished, whereas the synthetic Ptil variants accumulated to similar levels as with the hpBti9 construct, indicating the synthetic Ptil genes are not targeted by the hpPtil fragment (Fig. 5B). We then transiently expressed synPtila and synPtilb in leaves of single-copy hpPtil tomato plants; the gene encoding yellow fluorescent protein (YFP) was expressed in hpPtil and azygous plants as negative and positive control, respectively (Fig. 5C).
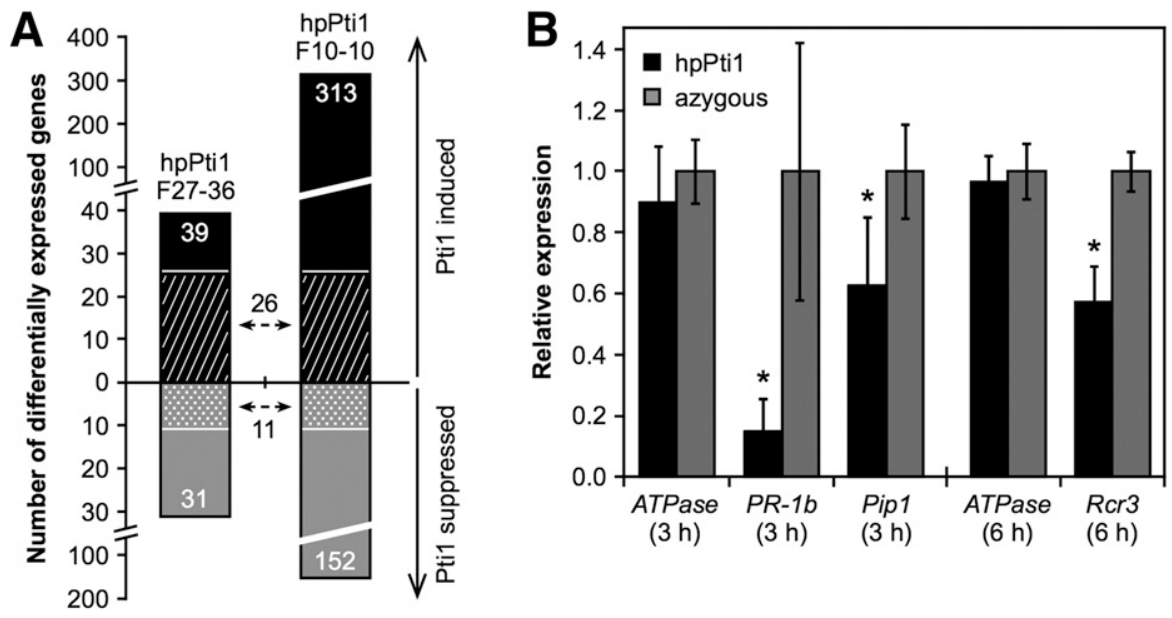

\begin{tabular}{llrrr}
$\mathbf{C}$ & & & & \\
Group & Go term & Group \% & Genome \% & $P$ value \\
\hline \multirow{2}{*}{$\begin{array}{l}\text { Pti1 induced } \\
\text { 26 genes) }\end{array}$} & Response to biotic stimulus & 57.69 & 16.73 & $1.42 \mathrm{E}-03$ \\
\cline { 2 - 5 } & Response to endogenous stimulus & 61.54 & 19.84 & $2.07 \mathrm{E}-03$ \\
\cline { 2 - 5 } & Response to other organism & 53.85 & 16.30 & $6.69 \mathrm{E}-03$ \\
\cline { 2 - 5 } & Defense response & 53.85 & 16.90 & $1.03 \mathrm{E}-02$ \\
\cline { 2 - 5 } & Response to hormone stimulus & 57.69 & 19.60 & $1.08 \mathrm{E}-02$ \\
\hline Pti1 suppressed & Regulation of carbohydrate biosynthetic process & 45.45 & 0.75 & $2.07 \mathrm{E}-06$ \\
\cline { 2 - 5 } (11 genes) & Regulation of carbohydrate metabolic process & 45.45 & 1.02 & $9.64 \mathrm{E}-06$ \\
\cline { 2 - 5 } & Regulation of glucosinolate biosynthetic process & 36.36 & 0.43 & $2.27 \mathrm{E}-05$ \\
\cline { 2 - 5 } & Regulation of sulfur metabolic process & 36.36 & 0.49 & $3.65 \mathrm{E}-05$ \\
\cline { 2 - 5 } & Sterol biosynthetic process & 45.45 & 1.78 & $1.52 \mathrm{E}-04$ \\
\hline
\end{tabular}

Fig. 3. Genes whose expression is induced by Pti1 after Pseudomonas syringae pv. tomato treatment are associated with pattern-triggered immunity. A, Number of genes whose expression is significantly induced or suppressed by Pti1 $(P \leq 0.05$, based on a false discovery rate correction) after treatment with DC3000_avrPto $a$ avrPtoB in either the single-copy hairpin-Pti1 (hpPti1) line (F27-36) or the multicopy hpPtil line (F10-10). Differentially expressed genes from the 3-h and 6-h datasets were combined for each line and duplicate genes were removed. The number of genes in common between the two lines is shown as patterned boxes (i.e., 26 induced and 11 suppressed). B, Transcript abundance of three genes with reduced expression in both hpPtil lines (i.e., genes that are normally induced by Pti1 after P. syringae pv. tomato treatment), i.e., PR- $1 b$ (Solyc00g174340) (Chen et al. 2014) and cysteine proteinase genes Pip1 (Solyc02g077040) and Rcr3 (Solyc02g076980) (Ilyas et al. 2015; Richau et al. 2012; Shindo et al. 2016). Expression levels were normalized to CBL1 (Pombo et al. 2014) and ATPase is shown as an internal control for each time point $(3$ or $6 \mathrm{~h})$. Bars show mean $\pm 95 \%$ confidence interval calculated from the RNA-Seq reads from the 3-h or 6-h dataset from the single-copy hpPti1 line (F27-36) compared with the azygous control line. Asterisks indicate significant differences in transcript abundance between hpPti1 and azygous control plants. $P$ values are 0.0027 for $P R-1 b, 0.0291$ for Pipl, and 0.0018 for Rcr3 (based on a false discovery rate correction). C, The genes shown in A that are in common between the two hpPti1 lines were subjected to a Gene Ontology (GO) term analysis. Shown are the top five GO terms for both the Pti1-induced genes and the Pti1-suppressed genes. The group percentage shows the frequency of a given GO term in the analyzed set of genes. The genome percentage shows the overall frequency of that GO term in the tomato genome. $P$ values are based on a false discovery rate correction. 
ROS production in response to flg22 increased in the presence of either synPtila or synPtilb, comparable to the level in the positive control (azygous plants transiently expressing YFP). Thus, the silencing of Ptila and Ptilb in the hpPtil lines and not silencing of nontarget genes leads to decreased ROS production.

Ptil proteins localize to the cell periphery and $S$-acylation on cysteine residues 6 and 7 is implicated in this localization.

Localization experiments of Pti1-like proteins in maize determined that ZmPtila localizes to the plasma membrane (PM)
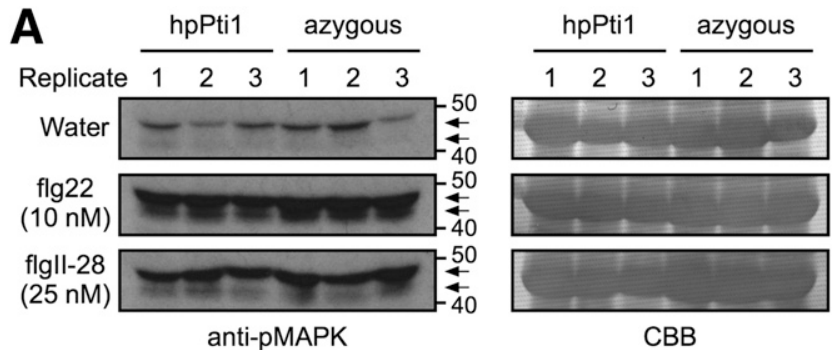

B
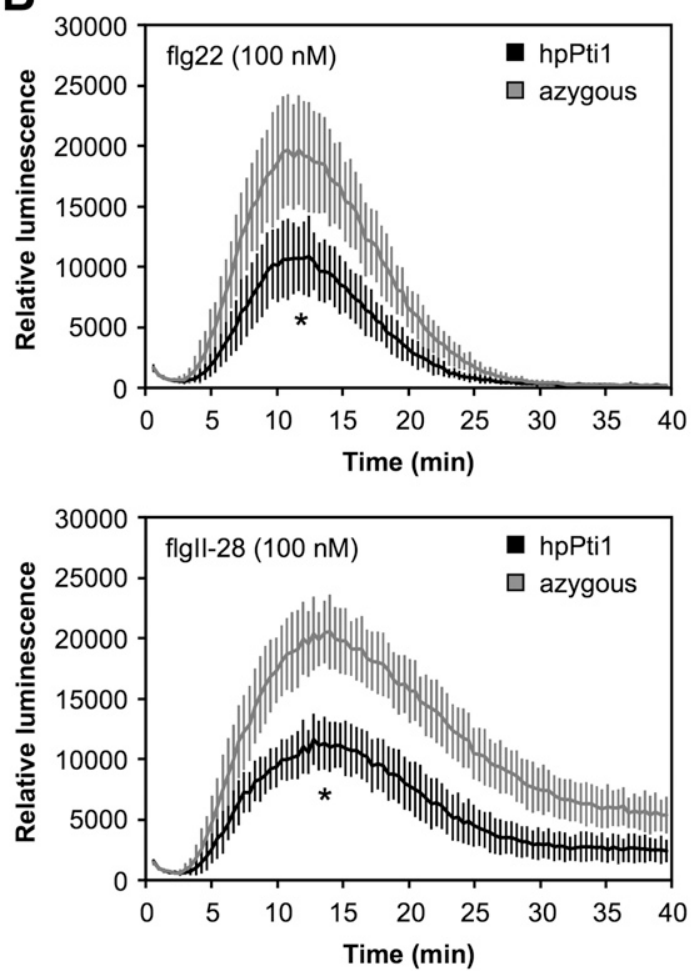

Fig. 4. Silencing of the Ptil genes in tomato diminishes the production of reactive oxygen species (ROS) in response to two flagellin-derived microbe-associated molecular patterns. A, Leaf discs from hairpin-Pti1 (hpPti1) or azygous plants were treated with water, $10 \mathrm{nM}$ flg22, or $25 \mathrm{nM}$ flgII-28 and were examined for mitogen-activated protein kinase (MAPK) activation. Protein was extracted from treated leaf discs 10 min after treatment and was subjected to immunoblotting, using an antibody (antipMAPK) that detects phosphorylated (activated) MAPKs. Shown are three biological replicates for each treatment, protein mass markers (40 and 50 $\mathrm{kDa}$ ), and arrows denoting the activated MAPKs. Coomassie brilliant blue (CBB) staining shows equal loading of protein. This experiment was performed twice with similar results. B, Leaf discs from hpPtil or azygous plants were treated with $100 \mathrm{nM}$ flg22 (top) or $100 \mathrm{nM}$ flgII-28 (bottom) and production of ROS was measured, using a chemiluminescence-based assay. Graphs show mean $\pm 99 \%$ confidence interval of seven plants per genotype. Asterisks indicate significant differences in the calculated areas under the curve between hpPtil and azygous control plants, based on a Student's $t$ test. $P$ values are 0.0004 for flg22 and $<0.0001$ for flgII-28. This experiment was performed three times with similar results. in onion epidermal cells, whereas an N-terminal truncation missing the first 20 amino acids was diffusely localized throughout the cell (Herrmann et al. 2006). Similarly, substitutions of glycine 2 as well as the cysteines at positions 3, 6, and 7 abolished PM localization of ZmPtila, consistent with putative myristoylation and $S$-acylation, respectively, of these residues (Boyle and Martin 2015; Herrmann et al. 2006). More recently, the rice Pti1 ortholog OsPtila was shown to localize to the PM in rice protoplasts and this localization was dependent on the predicted $S$-acylation sites C6 and C7 (Matsui et al. 2014).

The C6 and C7 residues are conserved in the tomato Ptil proteins and we introduced serine substitutions to test their importance in subcellular localization. We transiently expressed wild-type Ptila and Ptilb along with variants encoding C6S/C7S substitutions fused to $Y F P$ in leaves of $N$. benthamiana (Supplementary Fig. S5). The wild-type proteins localized to the cell periphery, whereas the $\mathrm{C} 6 \mathrm{~S} / \mathrm{C} 7 \mathrm{~S}$ mutants showed a more diffuse localization with obvious accumulation in the nuclei. YFP, included as a control, also accumulated in the nucleus in addition to the cytoplasm. Because the $\mathrm{C} 6$ and $\mathrm{C} 7$ residues are predicted $S$-acylation sites, it is possible that the peripheral localization of wild-type Ptil is due to $S$-acylation. Tomato Ptila was previously reported to have a diffuse localization when transiently expressed in onion epidermal cells and this observed discrepancy might be due to differences in the experimental system (Herrmann et al. 2006).

\section{Neither Ptila nor Ptilb play a demonstrable role in Pto-mediated resistance} to $P$. syringae pv. tomato in tomato.

Ptila was originally discovered in a yeast two-hybrid screen as an interactor of Pto and was thought to function in Pto-mediated resistance (Zhou et al. 1995). In light of our finding that one or both of the Pti1 kinases play a role in PTI, we used our hpPtil line to examine a possible role of these kinases in the Pto resistance pathway in tomato. Recognition of AvrPto in tomato requires both Pto and the NB-LRR protein Prf (Martin et al. 1993; Salmeron et al. 1994, 1996; Scofield et al. 1996; Tang et al. 1996). The hpPti1 line was made in the susceptible RG-prf3 background, which is homozygous for Pto but has a deletion in Prf, rendering the gene nonfunctional (Salmeron et al. 1996). Therefore, we crossed RG-PtoR (homozygous for both Pto and Prf) with hpPti1 plants to obtain $\mathrm{F}_{1}$ plants that contain a single functional copy of Prf, one copy of the hpPtil- silencing fragment, and two copies of Pto. As a control, we crossed RG-PtoR with RG-prf3 plants, which resulted in $F_{1}$ plants identical to the RG-PtoR $\times$ hpPti1 cross, except that no hpPtil construct is present.

The $F_{1}$ plants were inoculated with DC3000 (expressing avrPto and $a v r P t o B)$ at 2 titers $\left(10^{5}\right.$ and $\left.10^{6} \mathrm{CFU} / \mathrm{ml}\right)$, and disease symptoms were documented and bacterial populations were measured on days 3 and 2, respectively. No difference in Pto-mediated resistance was observed between the $F_{1}$ plants either carrying or lacking the hpPtil construct (Fig. 6A and B). At the lower inoculum concentration, all of the plants exhibited strong Pto-Prf-mediated resistance to $P$. syringae pv. tomato whereas, as expected, at the higher inoculum concentration, the plants carrying only a single copy of $\operatorname{Prf}$ developed mild disease symptoms and showed increased bacterial growth since PtoPrf-mediated resistance is known to be semidominant (Carland and Staskawicz 1993) (Fig. 6A and B).

It is possible that residual Ptil protein is present due to incomplete silencing and is sufficient to fully activate the Pto-Prf pathway. Therefore, we performed a second set of inoculations, using the same bacterial concentrations with resistant RG-PtoR plants and a transgenic line containing an RNAi construct that silences Pto in the RG-PtoR background (hpPto) (Pascuzzi 2006). RG-PtoR has two copies of Pto and Prf and showed no signs 
of disease at either inoculum level (Fig. 6A). The hpPto plants were highly susceptible, showing that silencing a gene directly involved in the Pto pathway leads to a complete loss of Pto-Prfmediated resistance even when two copies of Pto and Prf are present (Fig. 6A).

The questions remained whether a single copy of the hpPtil fragment is sufficient to effectively silence the two Ptil genes and whether the level of silencing is comparable to silencing of Pto by the hpPto construct. To address these issues, we first compared Ptil transcript abundance in RG-PtoR $\times$ hpPtil $F_{1}$ plants and the transgenic hpPtil line and found that there is no significant difference in silencing efficacy, as both lines have reduced Ptila and Ptilb mRNA levels of about 30\% compared with the RG-PtoR $\times$ RG-prf3 control $F_{1}$ plants (Fig. 6C). Second, we compared the hpPto plants to RG-PtoR plants and found that transcript abundance of Pto was reduced to about $10 \%$ in the presence of the silencing fragment (Supplementary Fig. S6). In conclusion, although Ptil transcripts are reduced to $30 \%$ of wildtype levels in the RG-PtoR $\times$ hpPtil $F_{1}$ plants, we see no effect on Pto-Prf-mediated resistance, indicating that neither Ptila nor Ptilb demonstrably contribute to this ETI response.

\section{DISCUSSION}

We have found that transgenic tomato plants silenced for Ptil are more susceptible to infection by $P$. syringae pv. tomato, fail to induce the expression of several defense-related genes associated with the PTI response, and have reduced ROS production in response to flagellin-derived peptides. Collectively, these observations support a role for Pti1 in PTI. Here, we place our findings in the context of previous work on Pti1 and Pti1related genes and discuss possible mechanisms by which the tomato Pti1 kinase might contribute to PTI. We also speculate on why Pti1 was originally identified as an interactor of the Pto kinase.

Our data support a role for Pti1 in the PTI signaling pathway in response to flagellin perception and indicate it acts either as part of the FLS2 and FLS3 receptor complexes or downstream of these complexes. Silencing of Ptila and Ptilb affected ROS production in response to flg 22 and flgII-28 and had a limited effect on the transcriptome but did not impact MAPK activation. The mechanism by which Ptil contributes to ROS production is unknown, but it is possible that it regulates the NADPH oxidase $\mathrm{RBOHD}$ (RBOHB in tobacco), which is localized to the plasma membrane and has a well-studied role in immunity-associated ROS generation (Adachi et al. 2015; Li et al. 2014). The Pti1 proteins are localized to the cell periphery, which places them in a position to be physically associated with the FLS2 and FLS3 receptor complexes or with an $\mathrm{RBOH}$ protein. In Arabidopsis, RBOHD is activated by direct $\mathrm{Ca}^{2+}$ binding and by phosphorylation by calcium-dependent protein kinases and BIK1 on partially overlapping residues (Boudsocq et al. 2010; Dubiella et al. 2013; Kadota et al. 2015). In N. benthamiana, RBOHB is required for flagellin-induced ROS production (Segonzac et al. 2011). In the future, we will test the hypothesis that the Ptil kinase might phosphorylate RBOHB and thereby contribute to its activation and ROS production.

A large number of genes are differentially expressed during the host response to individual MAMPs or to P. syringae pv. tomato (Pombo et al. 2014; Rosli et al. 2013). It is striking, then, that, despite the role of Ptil in enhancing PTI against $P$. syringae pv. tomato, our RNA-Seq analysis indicated the kinase plays a minor role in inducing gene expression in response to inoculation with $P$. syringae pv. tomato. Immunity-associated gene expression

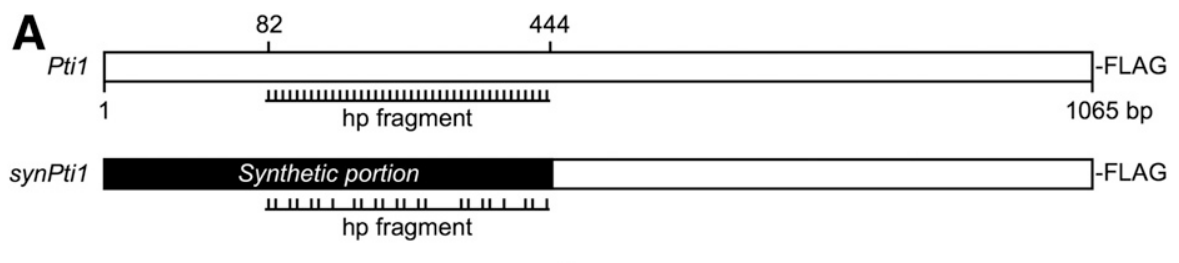

B

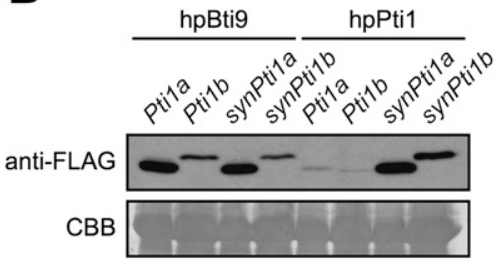

C

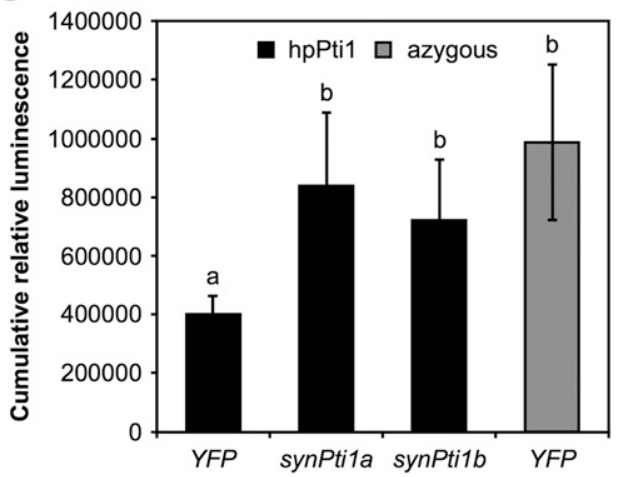

Fig. 5. Synthetic Ptil genes restore reactive oxygen species (ROS) production in hairpin-Pti1 (hpPti1) plants in response to flg22. A, A schematic illustration showing where the hairpin fragment anneals to the wild-type Ptil transcript. Synthetic versions of the tomato Ptil genes, referred to as synPtila and synPtilb, were made by synthesizing the $5^{\prime}$ portion of each gene with alternate codons (black) so that the hairpin fragment is no longer able to anneal. This synthetic portion was then fused to the $3^{\prime}$ wild-type sequence (white) by polymerase chain reaction. The synPtil genes encode the same amino acid sequences as the wild-type Ptil genes and all constructs included a FLAG tag at the C-terminus. B, Wild-type and synthetic versions of Ptila and Ptilb were transiently expressed in Nicotiana benthamiana leaves, using agroinfiltration together with either the hpPti1 fragment or the unrelated hpBti9 fragment (Zeng et al. 2011). Tissue samples were collected 2 days after infiltration, total protein was extracted, and Pti1 proteins were detected by anti-FLAG immunoblotting. Coomassie brilliant blue staining (CBB) shows equal loading of protein. C, Agroinfiltration was used to transiently express synPtila and synPtilb in leaves of hpPti1 tomato plants along with the yellow fluorescent protein gene (YFP) (negative control). Azygous plants transiently expressing $Y F P$ served as a positive control. Leaf discs were treated with $100 \mathrm{nM}$ flg22 and production of ROS was measured. The graph shows the cumulative relative luminescence (area under the curve). Bars show mean $\pm 99 \%$ confidence interval of eight plants per group. Different letters indicate significant differences based on a one-way analysis of variance, followed by Tukey's honest significant difference post hoc test. $P$ values are 0.0030 for hpPti1 + synPtila versus hpPti $1+Y F P, 0.0375$ for hpPti1 + synPtil $b$ versus hpPti1 $+Y F P$, and 0.0002 for hpPti1 $+Y F P$ versus azygous $+Y F P$. This experiment was performed twice with similar results. 
changes are typically regulated by MAPK cascades (Meng and Zhang 2013). We found no evidence for an effect of Ptil on MAPK activation, although it is possible small changes do occur and are below the sensitivity of our pMAPK assay. It is, perhaps, more likely that another signaling pathway is affected in the hpPti1 plants, possibly one normally triggered by ROS production (Apel and Hirt 2004). It is interesting that one of the genes most highly induced by Ptil is $P R-1 b$, which was recently shown to encode a propeptide, with its cleaved peptide, CAPE1, activating defense responses enhancing resistance to $P$. syringae pv. tomato (Chen et al. 2014). It is possible that perception of flagellin results in Ptil activation, which, in turn, leads to $P R-1 b$ expression through an unknown mechanism, and the resulting CAPE1 peptide further stimulates plant defense responses. Also of interest, our RNA-Seq data shows that the expression of two genes encoding cathepsin L-like cysteine proteinases, Rcr3 and Pip1, is induced by Ptil. It has been reported recently that the secreted $P$. syringae pv. tomato protein Cip1 (C14-inhibiting protein-1)
A

RG-PtoR x RG-prf3
Pto/Pto
Prf/-

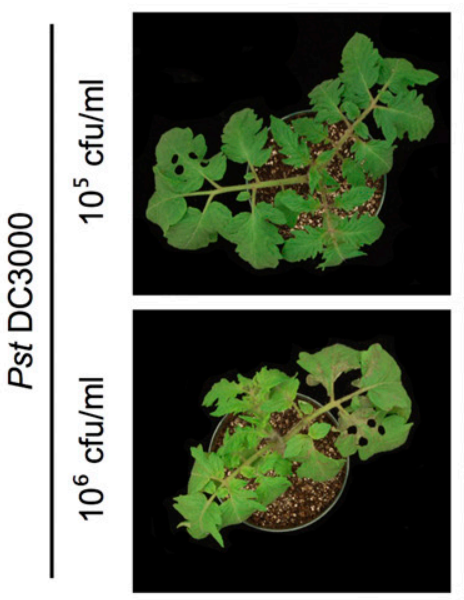

B

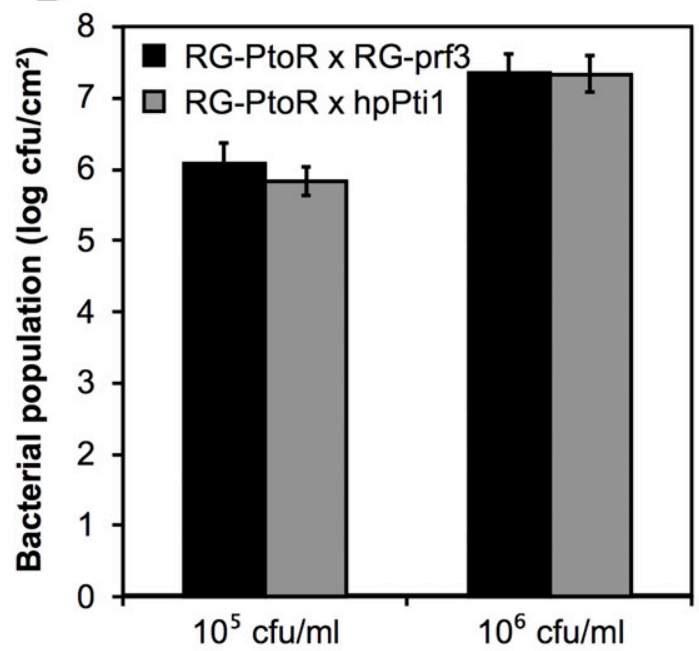

RG-PtoR x hpPti1
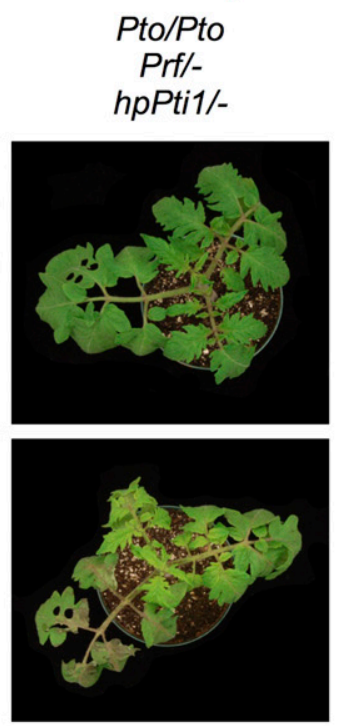

\section{RG-PtoR \\ Pto/Pto \\ Prf/Prf}
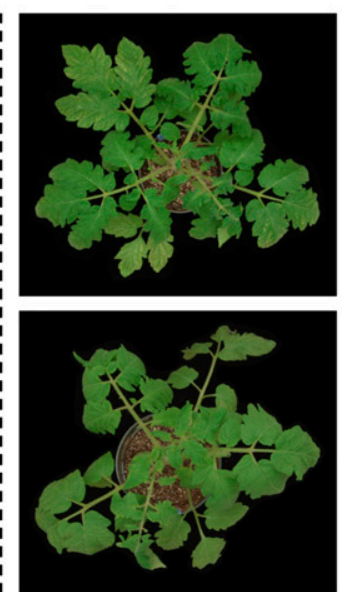

hpPto

Pto/Pto

Prf/Prf

hpPto/hpPto
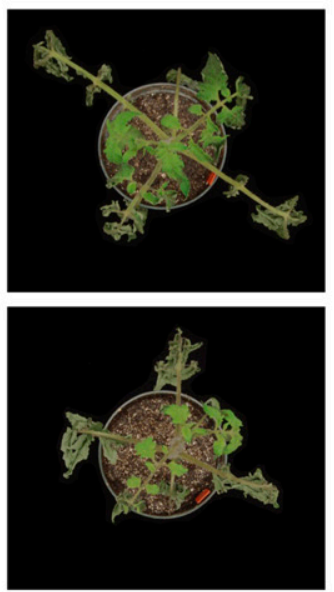

\section{C}

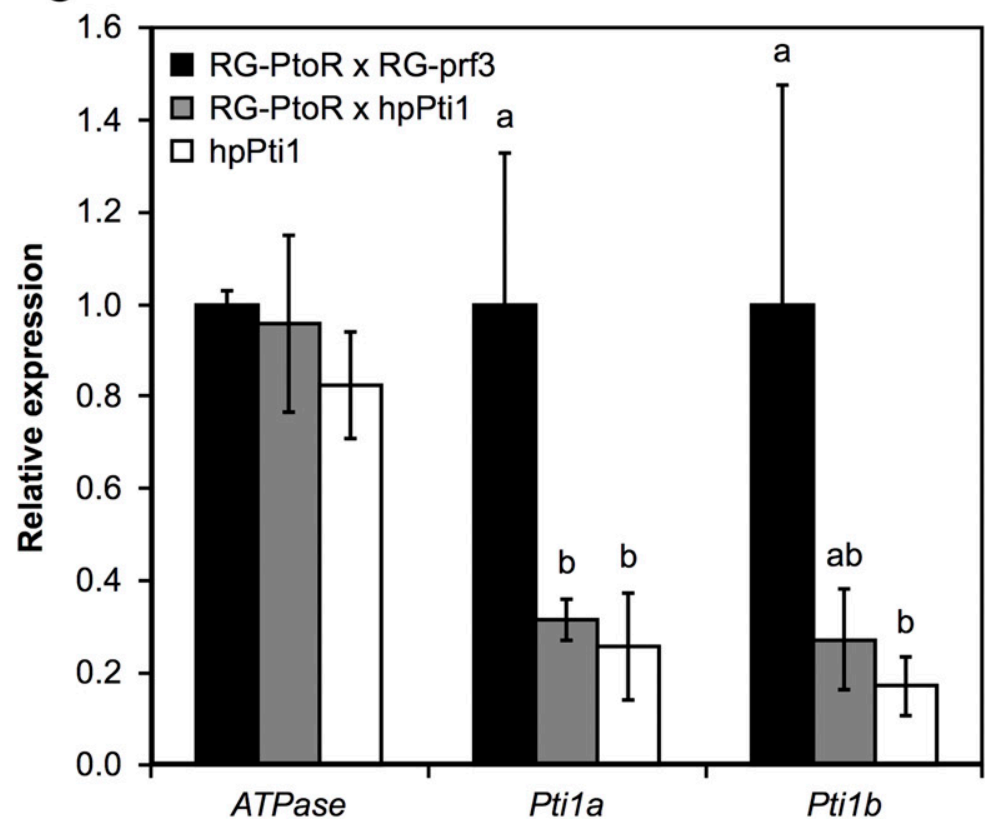

Fig. 6. Pto-mediated resistance is not detectably impaired in hairpin-Pti1 (hpPti1) plants. A, Four-week-old RG-PtoR $\times$ hpPti1 and RG-PtoR $\times$ RG-prf3 F plants were vacuum-infiltrated with DC3000 at $10^{5}$ or $10^{6} \mathrm{CFU} / \mathrm{ml}$ and disease symptoms were monitored. RG-PtoR and hpPto plants were included as resistant and susceptible controls, respectively. Photographs were taken 3 days after infiltration. Experiments involving RG-PtoR $F_{1}$ plants used four plants per genotype, and the RG-PtoR and hpPto experiments used three plants each. The dashed line indicates experiments performed on different days. B, Bacterial populations were determined in the plants used in A. Tissue samples were taken 2 days after infiltration. Bars show mean $\pm 99 \%$ confidence interval. No significant difference was found in either treatment. This experiment was performed twice with similar results. C, Quantitative polymerase chain reaction to monitor transcript levels of Ptila and Ptilb in the $\mathrm{F}_{1}$ plants. Expression data were normalized to CBL1 (Pombo et al. 2014) and are shown in relation to the RGPtoR $\times$ RG-prf3 control group. ATPase is shown as an internal control. Bars show mean $\pm 95 \%$ confidence interval of four plants per group. Homozygous hpPti1 plants were included for comparison. Different letters indicate significant differences based on a Brown-Forsythe test, followed by a Games-Howell post hoc test. $P$ values for Ptila are 0.024 for RG-PtoR $\times$ RG-prf3 versus RG-PtoR $\times$ hpPti 1 and 0.014 for RG-PtoR $\times$ RG-prf3 versus hpPti1, and $P$ values for $P$ ti $1 b$ are 0.054 for RG-PtoR $\times$ RG-prf3 versus RG-PtoR $\times$ hpPti1 and 0.040 for RG-PtoR $\times$ RG-prf3 versus hpPti1 
inhibits Rcr3 and Pip1 and a DC3000 $\Delta$ cipl mutant is less virulent on tomato, implicating these cysteine proteinases in plant immunity (Shindo et al. 2016).

Several studies have examined a role for the rice kinase OsPtila in resistance to the blast fungus Magnaporthe grisea as well as to Xanthomonas oryzae pv. oryzae, the causal agent of bacterial blight (Matsui et al. 2010a and b, 2014; Takahashi et al. 2007). Rice plants with transposon-induced mutations in OsPtila have a dwarf phenotype, form spontaneous lesions, show enhanced resistance to a compatible race of $M$. grisea and have increased $P R$ gene expression. These observations suggested that OsPtila negatively regulates PTI responses in rice. Overexpression of OsPtila led to lesion development (disease) by an incompatible race of $M$. grisea and enhanced lesion formation after inoculation with a compatible strain of $X$. oryzae pv. oryzae, indicating that OsPtila also represses ETI responses and further supported its role as a negative regulator of PTI (Takahashi et al. 2007). Silencing $R A R 1$, whose protein is a component of several $\mathrm{R}$ protein complexes (Shirasu 2009), negated the dwarf phenotype of rice ptila mutant plants and abolished both constitutive $P R$ gene expression and the enhanced lesion formation by the compatible race of $M$. grisea, thus showing that repression of immune signaling by OsPtila is dependent on RAR1 (Takahashi et al. 2007).

The results regarding OsPtila are in seeming conflict with our data showing a positive regulatory function for Ptil in the tomato PTI response. Despite the apparent opposite function of Ptil in rice and tomato, the two proteins function interchangeably in complementing the ptila rice plants (Takahashi et al. 2007). One proposed explanation for this discrepancy is that the signaling pathways downstream of Ptil have diverged in rice and tomato (Takahashi et al. 2007). Given the large evolutionary distance between the two species, this is a plausible hypothesis. However, an alternative explanation is that the lesion-mimic and dwarf phenotypes in ptila rice plants are due to the effects of an $\mathrm{R}$ protein that normally guards OsPtila. Absence of OsPtila in null mutants might trigger an ETI response that results in the observed autoimmune phenotypes. Such a scenario is reminiscent of RIN4 in Arabidopsis, which is guarded by two R proteins, RPM1 and RPS2 (Axtell and Staskawicz 2003; Mackey et al. 2002, 2003). RPS2 detects cleavage of RIN4 by the cognate $P$. syringae pv. tomato effector AvrRpt2 and activates ETI (Axtell and Staskawicz 2003; Mackey et al. 2003). Arabidopsis plants with a rin4 null mutation are seedling-lethal because of constitutive activation of RPS2, unless they also contain a rps 2 null mutation (Mackey et al. 2003). If Ptil does have a similar function in rice and tomato, then it is possible that the tomato variety we have used in our studies lacks an R protein that guards Ptil or that our hpPti1 plants are not null mutants and, therefore, the ETI response is not autoactivated, explaining why our hpPtil plants do not show stunted growth or autoimmune phenotypes. The $N$. benthamiana Ptil seems to be similar to tomato Ptil in having a positive effect on PTI and is, therefore, also divergent from rice in terms of downstream signaling events.

Arabidopsis has 11 Ptil-like kinases (Anthony et al. 2006; Liao et al. 2016), with the gene products of At1g48210, At1g48220, At2g47060 (PTI1-4), At3g17410, and At3g62220 being the closest to the tomato Ptil proteins. Of these five Ptil-like kinases, only PTI1-4 has been functionally characterized and found to interact with and be phosphorylated by the AGC kinase OXI1 (Forzani et al. 2011). PTI1-4 interacts with MPK6 and possibly MPK3 and both MAPKs phosphorylate OXI1 and PTI1-4 (Forzani et al. 2011). We have observed no interaction of Ptila with the tomato OXI1 ortholog in yeast (data not shown) and have no evidence that MAPKs function downstream of the Ptil proteins, as our hpPti1 tomato plants are unaffected in MAPK phosphorylation. Given these observations, there do not appear to be any obvious similarities between the characterized Arabidopsis Ptil-like kinases and tomato Ptila or Ptilb.

Previous work with transgenic tobacco plants overexpressing tomato Ptila reported faster development of PCD in response to P. syringae pv. tabaci expressing avrPto, compared with control plants (Zhou et al. 1995). This phenomenon was attributed to enhancement of the Pto resistance pathway due to overexpression of Ptila, whose protein was thought to function downstream of Pto (Zhou et al. 1995). However, later work did not support this finding when it was determined that, in tomato, Pto recognizes the $\mathrm{CD}$ loop in the core domain of AvrPto whereas, in tobacco, an unknown $\mathrm{R}$ protein recognizes the phosphorylated $\mathrm{C}$-terminal domain (CTD) of the effector (Anderson et al. 2006; Xing et al. 2007; Yeam et al. 2010). There are at least two explanations for the observation that Ptila overexpression appeared to enhance the ETI response in tobacco. First, Ptila may function downstream of the unknown $\mathrm{R}$ protein in tobacco that recognizes the AvrPto CTD. Second, the overexpression of tomato Ptila in tobacco may have had a 'dominant-negative' effect that interfered with the PTI response. Interference with PTI leads to enhanced delivery of effectors into the plant cell and faster PCD (Crabill et al. 2010; Oh et al. 2010). We do not currently have evidence to support or refute either of these possibilities. Although it might be interesting to further study the enhanced PCD response in Ptila-transgenic tobacco lines, tomato is better suited to study Pto-mediated ETI and our RG-PtoR $\times$ hpPtil and RG-PtoR $\times$ RG-prf3 crosses indicated that Ptil is not required for the ETI response activated in tomato upon recognition of AvrPto or AvrPtoB. This is consistent with previous observations that also did not support a role for Pti1 in the Pto pathway in tomato (Wu et al. 2004; Xiao et al. 2003).

A possible explanation for why Ptil was initially found to interact with Pto is that the latter kinase might have evolved as a 'decoy' of a host protein that plays a role in PTI by interacting with Pti1. A search of the predicted tomato proteome identified two malectin-like receptor kinases, Mal1 and Mal2 (Solyc11g072910 and Solyc06g005230), whose kinase domains have the highest sequence similarity to Pto. Malectin-like receptor kinases (also called Catharanthus roseus receptor-like kinase1-like kinases [CrRLK1Ls]) have an extracellular region containing two subdomains with similarity to the carbohydrate-binding domain of the animal malectin protein, a transmembrane domain, and an intracellular kinase domain (Lindner et al. 2012). One wellstudied CrRLK1L in Arabidopsis is FERONIA (FER) (At3g51550), which plays a role in cell-to-cell communication during pollination but which also impacts host responses to certain bacterial and fungal pathogens (Keinath et al. 2010; Kessler et al. 2010; Masachis et al. 2016). Interestingly, FER becomes rapidly phosphorylated upon treatment of tissue with flg22 and it accumulates along with FLS2 in PM 'rafts' during PTI (Benschop et al. 2007; Keinath et al. 2010). At early time points, Arabidopsis fer mutants support slightly increased bacterial growth; however, at later time points, fer plants allowed less bacterial proliferation than wildtype plants (Keinath et al. 2010). Based on these results, it was hypothesized that FER may act with FLS2 (Keinath et al. 2010). Indeed, it was reported recently that, in Arabidopsis, FER promotes ROS production in response to flg22, associates with both FLS2 and BAK1, and plays a positive role in regulating PTI (Stegmann et al. 2017). We are currently investigating whether Mal1 and Mal2 play a role in PTI in tomato and, if they do, whether Pti1 proteins might facilitate their function.

\section{MATERIALS AND METHODS}

\section{Plant material.}

Nicotiana benthamiana accession Nb-1 (Bombarely et al. 2012) was grown for 4 to 6 weeks in a controlled environment chamber with $16 \mathrm{~h}$ of light and $65 \%$ relative humidity, with 
temperatures of $24^{\circ} \mathrm{C}$ during light and $22^{\circ} \mathrm{C}$ during dark periods. The hpPtil lines were generated by cloning a segment of the tomato Ptila gene sequence (Fig. 2A) into pHELLSGATE8 (Helliwell et al. 2002) to obtain the hpPtil silencing construct. Tomato RG-prf3 (Salmeron et al. 1996) plants were transformed by the Center for Plant Biotechnology Research at the Boyce Thompson Institute (Ithaca, NY, U.S.A.). We obtained one single-copy line (F27-36) and one multicopy line (F10-10) as well as two corresponding 'azygous' control lines that had lost one or more transgenes due to segregation. The hpPto line was made in the RG-PtoR background and was described previously (Pascuzzi 2006). All tomato plants were grown in a greenhouse without supplemental light for 4 to 5 weeks before use in pathogen assays or RNA-Seq experiments.

\section{DNA cloning.}

The constructs for VIGS were developed as previously described (Rosli et al. 2013). Suitable sequences were selected using the Sol Genomics Network (SGN) VIGS tool (FernandezPozo et al. 2015a) and were cloned into pCR8/GW/TOPO (Invitrogen), followed by recombination into pQ11 (Liu et al. 2002), using the LR Clonase II enzyme mix (Invitrogen), and transformation into Agrobacterium tumefaciens GV2260 (Hellens et al. 2000). Full-length tomato Ptil genes were PCR-amplified and ligated into the pJLSmart Gateway entry vector (Mathieu et al. 2007) as described (Mathieu et al. 2014). Synthetic Ptil versions were obtained by changing the codons of the $5^{\prime}$ gene portions to prevent binding of the hpPtil fragment without altering the amino acid sequence. These synthetic portions were ordered from Integrated DNA Technologies and were fused to the wild-type 3' sequences by PCR and were cloned into pJLSmart. Cysteine-toserine substitutions were introduced, by PCR using complementary custom DNA oligonucleotides (Integrated DNA Technologies), following standard protocols. All Ptil constructs were recombined into the binary plant expression vectors pGWB411 or pGWB541 (Nakagawa et al. 2007), using the LR Clonase II enzyme mix to obtain C-terminal FLAG and enhanced YFP fusions, respectively. These expression constructs were transformed into A. tumefaciens strains 1D1249 (for tomato) and GV3101 (for N. benthamiana) (Hellens et al. 2000; Wroblewski et al. 2005). Vectors and constructs are shown in Supplementary Table S3.

\section{Cell death suppression assay.}

The cell death suppression assay in silenced $N$. benthamiana plants was performed as described previously (Chakravarthy et al. 2010; Rosli et al. 2013). Briefly, seedlings were syringeinfiltrated with Agrobacterium strains carrying the appropriate VIGS constructs and the assay was performed 6 weeks later. Nonpathogenic Pseudomonas fluorescens 55 (optical density at $600 \mathrm{~nm}\left[\mathrm{OD}_{600}\right]=0.5$ ) was syringe-infiltrated to induce PTI responses, followed $7 \mathrm{~h}$ later by infiltration of virulent $P$. syringae pv. tomato DC3000AhopQ1-1 $\left(\mathrm{OD}_{600}=0.01\right)$ (Wei et al. 2007) in overlapping circles. Disease symptoms were monitored and scored and were photographed 5 days later.

\section{Bacterial infection assay.}

Four-week-old tomato hpPti1 plants along with azygous and RG-prf3 controls were vacuum-infiltrated with DC3000 $\Delta a v r P t o \Delta a v r P t o B$ (Lin and Martin 2005) at $5 \times 10^{4}$ $\mathrm{CFU} / \mathrm{ml}$, as described previously (Anderson et al. 2006; Zeng et al. 2011). Inoculated plants were kept in a controlled environment chamber and bacterial populations were assessed by taking leaf samples a few hours after infiltration and 2 days later. Plants were photographed 5 days after infiltration to document disease symptoms. The RG-PtoR $\mathrm{F}_{1}$ plants along with RG-PtoR and hpPto controls were vacuum-infiltrated with DC3000 at $10^{5}$ or $10^{6} \mathrm{CFU} / \mathrm{ml}$. Photographs were taken 3 days after infiltration.

\section{MAPK phosphorylation assay.}

Leaf discs of tomato hpPtil and azygous control plants were floated in water for $1 \mathrm{~h}$ to let the wound response subside. The water was replaced by fresh water containing $10 \mathrm{nM}$ flg22 (GenScript), $25 \mathrm{nM}$ flgII-28 (EZBiolab), or no peptide (negative control), the leaf discs were incubated for $10 \mathrm{~min}$ and were frozen and ground in liquid nitrogen. Whole protein was extracted, using a buffer containing $10 \%$ glycerol, $25 \mathrm{mM}$ Tris- $\mathrm{HCl}(\mathrm{pH} 8.0)$, $1 \mathrm{mM}$ EDTA (pH 8.0), $150 \mathrm{mM} \mathrm{NaCl}, 10 \mathrm{mM}$ dithiothreitol (American Bioanalytical), and 0.15\% IGEPAL CA-630 (Nonidet P-40; Sigma-Aldrich), with cOmplete ULTRA EDTA-free protease inhibitor (Roche Diagnostics) and PhosSTOP phosphatase inhibitor (Roche Diagnostics). Samples were incubated for $15 \mathrm{~min}$ at $4^{\circ} \mathrm{C}$, the supernatants were collected and boiled in Laemmli sample buffer. Gel electrophoresis and immunoblotting was performed following standard protocols. MAPK phosphorylation was detected using the phospho-p44/42 MAPK (Erk1/2) antibody (antipMAPK; Cell Signaling), following the manufacturer's instructions.

\section{ROS production assay.}

Production of ROS was measured as described previously (Chakravarthy et al. 2010; Clarke et al. 2013), with modifications. Leaf discs of tomato hpPti1 and azygous control plants were floated overnight in water in white, flat-bottom, 96-well plates (Greiner Bio-One). The water was removed $12 \mathrm{~h}$ later and a solution containing $100 \mathrm{nM}$ flg22, $34 \mu \mathrm{g} / \mathrm{ml}$ luminol (Sigma-Aldrich), and $20 \mu \mathrm{g}$ of horseradish peroxidase per milliliter (type VI-A; Sigma-Aldrich) was added. ROS production was quantified by means of luminescence output from each well over time. Luminescence was measured using a Synergy 2 microplate reader (BioTek).

\section{Agrobacterium-mediated transient expression.}

Preparation of Agrobacterium strains and plant infiltrations were performed as described previously (Mathieu et al. 2014; Kraus et al. 2016). Briefly, confirmed Agrobacterium strains were grown on lysogeny broth plates with the appropriate antibiotics for 36 to $48 \mathrm{~h}$ at $30^{\circ} \mathrm{C}$. Cells were collected and suspended in infiltration buffer containing $10 \mathrm{mM} \mathrm{MgCl}_{2}, 10 \mathrm{mM}$ MES (pH 5.7), and $200 \mu \mathrm{M}$ acetosyringone (Sigma-Aldrich), the $\mathrm{OD}_{600}$ for each strain was adjusted to 0.3 , and cells were incubated for $1 \mathrm{~h}$ at room temperature. Leaves of $N$. benthamiana and tomato plants were infiltrated with needle-less syringes and the plants were placed in a controlled environment chamber (described above).

\section{Protein detection.}

Discs of Agrobacterium-transformed N. benthamiana leaf tissue were collected 2 days after agroinfiltration, were frozen, and were ground in liquid nitrogen. Proteins were extracted as described above, except that no phosphatase inhibitor was used for the protein extraction. FLAG-tagged proteins were detected using anti-FLAG-HRP (Sigma-Aldrich).

\section{Fluorescence imaging.}

Sections of $N$. benthamiana leaves expressing Ptil-YFP fusions were mounted on microscopy slides 2 days after agroinfiltration and were analyzed on a Leica DM5500 epifluorescence microscope. Images were acquired with a Retiga 2000R CCD camera (QImaging) using QCapture Pro software (QImaging).

\section{qPCR.}

Total RNA was extracted from $N$. benthamiana and tomato leaf tissue, using Plant RNA purification reagent (Invitrogen) according to the manufacturer's instructions. RNA samples were additionally purified using RNeasy Mini columns (Qiagen) 
and the isolated RNA was treated with RNase-free TURBO DNase (Ambion), following the respective manufacturers' protocols. First-strand cDNA synthesis and qPCR were performed exactly as described previously (Breuillin-Sessoms et al. 2015). Cycle numbers of each plant were normalized ( $\triangle \mathrm{CT}$ ) to PP2A (Liu et al. 2012) for $N$. benthamiana and $C B L 1$ (Pombo et al. 2014) for tomato. Means and confidence intervals of the transformed cycle numbers $\left(2^{\Delta \mathrm{CT}}\right)$ of the biological replicates were calculated and normalized to the control plants (EC1 for $N$. benthamiana; RG-PtoR $\times$ RG-prf3 $\mathrm{F}_{1}$ and RG-PtoR for tomato). The qPCR oligonucleotides used are given in Supplementary Table S4.

\section{RNA sequencing analysis.}

Tomato hpPti1 lines F27-36 (single hpPti1 copy) and F10-10 (multiple hpPti1 copies) along with an azygous control line were vacuum-infiltrated with DC3000 $\Delta a v r P t o \Delta a v r P t o B$ at $5 \times$ $10^{4} \mathrm{CFU} / \mathrm{ml}$. Tissue samples were taken 3 and $6 \mathrm{~h}$ after infiltration. The treatments were repeated in three successive weeks (three biological replicates). RNA isolation, library preparation, and RNA-Seq analysis were performed using tomato genome sequence version 2.40 as described previously (Rosli et al. 2013). The chosen cutoffs for differentially regulated genes were $\geq 3$ RPKM in at least one of the treatments, $\geq 1.5$-fold expression change, and $P \leq 0.05$. To capture the number of differentially expressed genes in the two hpPti1 lines, the 3- and 6-h datasets were combined for each hpPtil line and duplicate genes were removed. The RNA-Seq reads for visualizing Ptila and Ptilb silencing were taken from the 6-h dataset, were normalized to $C B L 1$ (Pombo et al. 2014), and were expressed in relation to the azygous control line. The PTI 'marker genes' were visualized the same way, based on the 3-h dataset. The Ptila and Ptilb expression data in response to different PTI inducers (Rosli et al. 2013) were simply visualized in graph form.

\section{Phylogenetic analysis.}

Coding sequences for the Ptil genes from tomato and $N$. benthamiana were obtained from SGN (Fernandez-Pozo et al. 2015b). Alignment and tree construction were performed with MEGA7, with the guidance of a step-by-step protocol (Hall 2013; Kumar et al. 2016). Specifically, DNA sequences were aligned using the MUSCLE method (align codons, default settings). Maximum likelihood (ML) substitution models were predicted using the default settings. The phylogenetic tree was estimated using the ML method (Tamura 3-parameter model, gamma distributed rates among sites, partial deletion of gaps/missing data, default settings otherwise). Reliability of the tree was estimated using the bootstrap method (1,000 replicates). The alignment file is provided as Supplementary Dataset S3.

\section{Gene sequences.}

Gene sequences are available from the SGN database under the following accession numbers: SlPtila (Solyc12g098980), SlPtilb (Solyc05g053230), NbPtila (Niben101Scf01236g02003), NbPtilb (Niben101Scf01334g04008), NbPtilc (Niben101Scf01671g04002), and NbPtild (Niben101Scf01820g00026). RNA-Seq reads have been deposited in the National Center for Biotechnology Information Sequence Read Archive under accession number SRP076863, and analyzed data are available from the Tomato Functional Genomics database under accession number D014.

\section{ACKNOWLEDGMENTS}

We thank D. S. Floss for help with the qPCR experiments, H. G. Rosli for advice on RNA-Seq data analysis, H. L. McLane for developing the hpPti1 construct, P. L. Reeves for plant care, and S. R. Hind and F. Giska for helpful comments on the manuscript. This work was supported, in part, by
National Science Foundation grants IOS-1025642, IOS-1451754, and IOS1546625 (G. B. Martin).

\section{LITERATURE CITED}

Adachi, H., Nakano, T., Miyagawa, N., Ishihama, N., Yoshioka, M., Katou, Y., Yaeno, T., Shirasu, K., and Yoshioka, H. 2015. WRKY transcription factors phosphorylated by MAPK regulate a plant immune NADPH oxidase in Nicotiana benthamiana. Plant Cell 27:2645-2663.

Anderson, J. C., Pascuzzi, P. E., Xiao, F., Sessa, G., and Martin, G. B. 2006. Host-mediated phosphorylation of type III effector AvrPto promotes Pseudomonas virulence and avirulence in tomato. Plant Cell 18:502-514.

Anthony, R. G., Khan, S., Costa, J., Pais, M. S., and Bögre, L. 2006. The Arabidopsis protein kinase PTI1-2 is activated by convergent phosphatidic acid and oxidative stress signaling pathways downstream of PDK1 and OXI1. J. Biol. Chem. 281:37536-37546.

Apel, K., and Hirt, H. 2004. Reactive oxygen species: Metabolism, oxidative stress, and signal transduction. Annu. Rev. Plant Biol. 55: 373-399.

Axtell, M. J., and Staskawicz, B. J. 2003. Initiation of RPS2-specified disease resistance in Arabidopsis is coupled to the AvrRpt2-directed elimination of RIN4. Cell 112:369-377.

Benschop, J. J., Mohammed, S., O’Flaherty, M., Heck, A. J., Slijper, M., and Menke, F. L. 2007. Quantitative phosphoproteomics of early elicitor signaling in Arabidopsis. Mol. Cell. Proteomics 6:1198-1214.

Bent, A. F., and Mackey, D. 2007. Elicitors, effectors, and $R$ genes: The new paradigm and a lifetime supply of questions. Annu. Rev. Phytopathol. 45:399-436.

Block, A., and Alfano, J. R. 2011. Plant targets for Pseudomonas syringae type III effectors: Virulence targets or guarded decoys? Curr. Opin. Microbiol. 14:39-46.

Boller, T., and Felix, G. 2009. A renaissance of elicitors: Perception of microbe-associated molecular patterns and danger signals by patternrecognition receptors. Annu. Rev. Plant Biol. 60:379-406.

Bombarely, A., Rosli, H. G., Vrebalov, J., Moffett, P., Mueller, L. A., and Martin, G. B. 2012. A draft genome sequence of Nicotiana benthamiana to enhance molecular plant-microbe biology research. Mol. PlantMicrobe Interact 25:1523-1530.

Boudsocq, M., Willmann, M. R., McCormack, M., Lee, H., Shan, L., He, P. Bush, J., Cheng, S. H., and Sheen, J. 2010. Differential innate immune signalling via $\mathrm{Ca}^{2+}$ sensor protein kinases. Nature 464:418-422.

Boyle, P. C., and Martin, G. B. 2015. Greasy tactics in the plant-pathogen molecular arms race. J. Exp. Bot. 66:1607-1616.

Breuillin-Sessoms, F., Floss, D. S., Gomez, S. K., Pumplin, N., Ding, Y., Levesque-Tremblay, V., Noar, R. D., Daniels, D. A., Bravo, A. Eaglesham, J. B., Benedito, V. A., Udvardi, M. K., and Harrison, M. J. 2015. Suppression of arbuscule degeneration in Medicago truncatula phosphate transporter4 mutants is dependent on the ammonium transporter 2 family protein AMT2;3. Plant Cell 27:1352-1366.

Buell, C. R., Joardar, V., Lindeberg, M., Selengut, J., Paulsen, I. T., Gwinn, M. L., Dodson, R. J., Deboy, R. T., Durkin, A. S., Kolonay, J. F., Madupu, R., Daugherty, S., Brinkac, L., Beanan, M. J., Haft, D. H., Nelson, W. C., Davidsen, T., Zafar, N., Zhou, L., Liu, J., Yuan, Q., Khouri, H., Fedorova, N., Tran, B., Russell, D., Berry, K., Utterback, T., Van Aken, S. E., Feldblyum, T. V., D'Ascenzo, M., Deng, W. L., Ramos, A. R., Alfano, J. R., Cartinhour, S., Chatterjee, A. K., Delaney, T. P., Lazarowitz, S. G., Martin, G. B., Schneider, D. J., Tang, X., Bender, C. L., White, O., Fraser, C. M., and Collmer, A. 2003. The complete genome sequence of the Arabidopsis and tomato pathogen Pseudomonas syringae pv. tomato DC3000. Proc. Natl. Acad. Sci. U.S.A. 100:10181-10186.

Cai, R., Lewis, J., Yan, S., Liu, H., Clarke, C. R., Campanile, F., Almeida, N. F., Studholme, D. J., Lindeberg, M., Schneider, D., Zaccardelli, M., Setubal, J. C., Morales-Lizcano, N. P., Bernal, A., Coaker, G., Baker, C., Bender, C. L., Leman, S., and Vinatzer, B. A. 2011. The plant pathogen Pseudomonas syringae pv. tomato is genetically monomorphic and under strong selection to evade tomato immunity. PLoS Pathog. 7: e1002130.

Carland, F. M., and Staskawicz, B. J. 1993. Genetic characterization of the Pto locus of tomato: Semi-dominance and cosegregation of resistance to Pseudomonas syringae pathovar tomato and sensitivity to the insecticide Fenthion. Mol. Gen. Genet. 239:17-27.

Chakravarthy, S., Velásquez, A. C., Ekengren, S. K., Collmer, A., and Martin, G. B. 2010. Identification of Nicotiana benthamiana genes involved in pathogen-associated molecular pattern-triggered immunity. Mol. Plant-Microbe Interact 23:715-726.

Chen, Y. L., Lee, C. Y., Cheng, K. T., Chang, W. H., Huang, R. N., Nam, H. G., and Chen, Y. R. 2014. Quantitative peptidomics study reveals that 
a wound-induced peptide from PR-1 regulates immune signaling in tomato. Plant Cell 26:4135-4148.

Cheng, W., Munkvold, K. R., Gao, H., Mathieu, J., Schwizer, S., Wang, S. Yan, Y. B., Wang, J., Martin, G. B., and Chai, J. 2011. Structural analysis of Pseudomonas syringae AvrPtoB bound to host BAK1 reveals two similar kinase-interacting domains in a type III Effector. Cell Host Microbe 10:616-626.

Chinchilla, D., Bauer, Z., Regenass, M., Boller, T., and Felix, G. 2006. The Arabidopsis receptor kinase FLS2 binds flg22 and determines the specificity of flagellin perception. Plant Cell 18:465-476.

Clarke, C. R., Chinchilla, D., Hind, S. R., Taguchi, F., Miki, R., Ichinose, Y. Martin, G. B., Leman, S., Felix, G., and Vinatzer, B. A. 2013. Allelic variation in two distinct Pseudomonas syringae flagellin epitopes modulates the strength of plant immune responses but not bacterial motility. New Phytol. 200:847-860.

Cook, D. E., Mesarich, C. H., and Thomma, B. P. 2015. Understanding plant immunity as a surveillance system to detect invasion. Annu. Rev. Phytopathol. 53:541-563.

Couto, D., and Zipfel, C. 2016. Regulation of pattern recognition receptor signalling in plants. Nat. Rev. Immunol. 16:537-552.

Crabill, E., Joe, A., Block, A., van Rooyen, J. M., and Alfano, J. R. 2010. Plant immunity directly or indirectly restricts the injection of type III effectors by the Pseudomonas syringae type III secretion system. Plant Physiol. 154:233-244.

Cunnac, S., Chakravarthy, S., Kvitko, B. H., Russell, A. B., Martin, G. B., and Collmer, A. 2011. Genetic disassembly and combinatorial reassembly identify a minimal functional repertoire of type III effectors in Pseudomonas syringae. Proc. Natl. Acad. Sci. U.S.A. 108:2975-2980.

Dodds, P. N., and Rathjen, J. P. 2010. Plant immunity: Towards an integrated view of plant-pathogen interactions. Nat. Rev. Genet. 11:539-548.

Dong, J., Xiao, F., Fan, F., Gu, L., Cang, H., Martin, G. B., and Chai, J 2009. Crystal structure of the complex between Pseudomonas effector AvrPtoB and the tomato Pto kinase reveals both a shared and a unique interface compared with AvrPto-Pto. Plant Cell 21:1846-1859.

Dou, D., and Zhou, J. M. 2012. Phytopathogen effectors subverting host immunity: Different foes, similar battleground. Cell Host Microbe 12: 484-495.

Dubiella, U., Seybold, H., Durian, G., Komander, E., Lassig, R., Witte, C. P., Schulze, W. X., and Romeis, T. 2013. Calcium-dependent protein kinase/NADPH oxidase activation circuit is required for rapid defense signal propagation. Proc. Natl. Acad. Sci. U.S.A. 110:8744-8749.

Felix, G., Duran, J. D., Volko, S., and Boller, T. 1999. Plants have a sensitive perception system for the most conserved domain of bacterial flagellin. Plant J. 18:265-276.

Fernandez-Pozo, N., Menda, N., Edwards, J. D., Saha, S., Tecle, I. Y., Strickler, S. R., Bombarely, A., Fisher-York, T., Pujar, A., Foerster, H., Yan, A., and Mueller, L. A. 2015b. The Sol Genomics Network (SGN)-From genotype to phenotype to breeding. Nucleic Acids Res. 43:D1036-D1041.

Fernandez-Pozo, N., Rosli, H. G., Martin, G. B., and Mueller, L. A. 2015a. The SGN VIGS tool: User-friendly software to design virus-induced gene silencing (VIGS) constructs for functional genomics. Mol. Plant 8: 486-488.

Forzani, C., Carreri, A., de la Fuente van Bentem, S., Lecourieux, D., Lecourieux, F., and Hirt, H. 2011. The Arabidopsis protein kinase Ptointeracting 1-4 is a common target of the oxidative signal-inducible 1 and mitogen-activated protein kinases. FEBS J. 278:1126-1136.

Gómez-Gómez, L., and Boller, T. 2000. FLS2: An LRR receptor-like kinase involved in the perception of the bacterial elicitor flagellin in Arabidopsis. Mol. Cell 5:1003-1011.

Hall, B. G. 2013. Building phylogenetic trees from molecular data with MEGA. Mol. Biol. Evol. 30:1229-1235.

He, P., Shan, L., Lin, N. C., Martin, G. B., Kemmerling, B., Nürnberger, T., and Sheen, J. 2006. Specific bacterial suppressors of MAMP signaling upstream of MAPKKK in Arabidopsis innate immunity. Cell 125:563-575.

Hellens, R., Mullineaux, P., and Klee, H. 2000. Technical Focus:a guide to Agrobacterium binary Ti vectors. Trends Plant Sci. 5:446-451.

Helliwell, C. A., Wesley, S. V., Wielopolska, A. J., and Waterhouse, P. M. 2002. High-throughput vectors for efficient gene silencing in plants. Funct. Plant Biol. 29:1217-1225.

Herrmann, M. M., Pinto, S., Kluth, J., Wienand, U., and Lorbiecke, R. 2006. The PTI1-like kinase ZmPtila from maize (Zea mays L.) co-localizes with callose at the plasma membrane of pollen and facilitates a competitive advantage to the male gametophyte. BMC Plant Biol. 6:22.

Hind, S. R., Strickler, S. R., Boyle, P. C., Dunham, D. M., Bao, Z., O’Doherty, I. M., Baccile, J. A., Hoki, J. S., Viox, E. G., Clarke, C. R., Vinatzer, B. A., Schroeder, F. C., and Martin, G. B. 2016. Tomato receptor FLAGELLIN-SENSING 3 binds flgII-28 and activates the plant immune system. Nat Plants 2:16128.
Hohmann, U., Lau, K., and Hothorn, M. 2017. The structural basis of ligand perception and signal activation by receptor kinases. Annu. Rev. Plant Biol. 68:109-137.

Ilyas, M., Hörger, A. C., Bozkurt, T. O., van den Burg, H. A., Kaschani, F., Kaiser, M., Belhaj, K., Smoker, M., Joosten, M. H., Kamoun, S., and van der Hoorn, R. A. 2015. Functional divergence of two secreted immune proteases of tomato. Curr. Biol. 25:2300-2306.

Jones, J. B. 1991. Bacterial speck. Pages 26-27 in: Compendium of tomato diseases. J. B. Jones, J. P. Jones, R. E. Stall, and T. A. Zitter, eds. APS Press, St. Paul, MN, U.S.A.

Jones, J. D., and Dangl, J. L. 2006. The plant immune system. Nature 444: 323-329.

Kadota, Y., Shirasu, K., and Zipfel, C. 2015. Regulation of the NADPH oxidase RBOHD during plant immunity. Plant Cell Physiol. 56: 1472-1480.

Keinath, N. F., Kierszniowska, S., Lorek, J., Bourdais, G., Kessler, S. A., Shimosato-Asano, H., Grossniklaus, U., Schulze, W. X., Robatzek, S., and Panstruga, R. 2010. PAMP (pathogen-associated molecular pattern)induced changes in plasma membrane compartmentalization revea novel components of plant immunity. J. Biol. Chem. 285:39140-39149.

Kessler, S. A., Shimosato-Asano, H., Keinath, N. F., Wuest, S. E., Ingram, G., Panstruga, R., and Grossniklaus, U. 2010. Conserved molecular components for pollen tube reception and fungal invasion. Science 330:968-971.

Kraus, C. M., Munkvold, K. R., and Martin, G. B. 2016. Natural variation in tomato reveals differences in the recognition of AvrPto and AvrPtoB effectors from Pseudomonas syringae. Mol. Plant 9:639-649.

Kumar, D., Gustafsson, C., and Klessig, D. F. 2006. Validation of RNAi silencing specificity using synthetic genes: Salicylic acid-binding protein 2 is required for innate immunity in plants. Plant J. 45:863-868.

Kumar, S., Stecher, G., and Tamura, K. 2016. MEGA7: Molecular evolutionary genetics analysis version 7.0 for bigger datasets. Mol. Biol. Evol. 33: 1870-1874.

Kvitko, B. H., Park, D. H., Velásquez, A. C., Wei, C. F., Russell, A. B., Martin, G. B., Schneider, D. J., and Collmer, A. 2009. Deletions in the repertoire of Pseudomonas syringae pv. tomato DC3000 type III secretion effector genes reveal functional overlap among effectors. PLoS Pathog. 5: e1000388.

Li, L., Li, M., Yu, L., Zhou, Z., Liang, X., Liu, Z., Cai, G., Gao, L., Zhang, X., Wang, Y., Chen, S., and Zhou, J. M. 2014. The FLS2-associated kinase BIK1 directly phosphorylates the NADPH oxidase RbohD to control plant immunity. Cell Host Microbe 15:329-338.

Liao, H.-Z., Zhu, M.-M., Cui, H.-H., Du, X.-Y., Tang, Y., Chen, L.-Q., Ye, D., and Zhang, X.-Q. 2016. MARIS plays important roles in Arabidopsis pollen tube and root hair growth. J. Integr. Plant Biol. 58:927-940.

Lin, N. C., and Martin, G. B. 2005. An avrPto/avrPtoB mutant of Pseudomonas syringae pv. tomato DC3000 does not elicit Pto-mediated resistance and is less virulent on tomato. Mol. Plant-Microbe Interact 18: 43-51.

Lindeberg, M., Cunnac, S., and Collmer, A. 2012. Pseudomonas syringae type III effector repertoires: Last words in endless arguments. Trends Microbiol. 20:199-208.

Lindner, H., Müller, L. M., Boisson-Dernier, A., and Grossniklaus, U. 2012. CrRLK1L receptor-like kinases: Not just another brick in the wall. Curr. Opin. Plant Biol. 15:659-669.

Liu, D., Shi, L., Han, C., Yu, J., Li, D., and Zhang, Y. 2012. Validation of reference genes for gene expression studies in virus-infected Nicotiana benthamiana using quantitative real-time PCR. PLoS ONE 7:e46451.

Liu, Y., Schiff, M., and Dinesh-Kumar, S. P. 2002. Virus-induced gene silencing in tomato. Plant J. 31:777-786.

Macho, A. P., and Zipfel, C. 2015. Targeting of plant pattern recognition receptor-triggered immunity by bacterial type-III secretion system effectors. Curr. Opin. Microbiol. 23:14-22.

Mackey, D., Belkhadir, Y., Alonso, J. M., Ecker, J. R., and Dangl, J. L. 2003. Arabidopsis RIN4 is a target of the type III virulence effector AvrRpt2 and modulates RPS2-mediated resistance. Cell 112:379-389.

Mackey, D., Holt, B. F., 3rd, Wiig, A., and Dangl, J. L. 2002. RIN4 interacts with Pseudomonas syringae type III effector molecules and is required for RPM1-mediated resistance in Arabidopsis. Cell 108:743-754.

Martin, G. B. 2012. Suppression and activation of the plant immune system by Pseudomonas syringae effectors AvrPto and AvrPtoB. Pages 123-154 in: Effectors in Plant-Microbe Interactions. F. Martin, and S. Kamoun, eds. Wiley-Blackwell, Oxford.

Martin, G. B., Brommonschenkel, S. H., Chunwongse, J., Frary, A., Ganal, M. W., Spivey, R., Wu, T., Earle, E. D., and Tanksley, S. D. 1993. Mapbased cloning of a protein kinase gene conferring disease resistance in tomato. Science 262:1432-1436.

Masachis, S., Segorbe, D., Turrà, D., Leon-Ruiz, M., Fürst, U., El Ghalid, M., Leonard, G., López-Berges, M. S., Richards, T. A., Felix, G., and Di 
Pietro, A. 2016. A fungal pathogen secretes plant alkalinizing peptides to increase infection. Nat. Microbiol. 1:16043.

Mathieu, J., Schwizer, S., and Martin, G. B. 2014. Pto kinase binds two domains of AvrPtoB and its proximity to the effector E3 ligase determines if it evades degradation and activates plant immunity. PLoS Pathog. 10:e1004227.

Mathieu, J., Warthmann, N., Küttner, F., and Schmid, M. 2007. Export of FT protein from phloem companion cells is sufficient for floral induction in Arabidopsis. Curr. Biol. 17:1055-1060.

Matsui, H., Fujiwara, M., Hamada, S., Shimamoto, K., Nomura, Y., Nakagami, H., Takahashi, A., and Hirochika, H. 2014. Plasma membrane localization is essential for Oryza sativa Pto-interacting protein 1a-mediated negative regulation of immune signaling in rice. Plant Physiol. 166:327-336.

Matsui, H., Miyao, A., Takahashi, A., and Hirochika, H. 2010a. Pdk1 kinase regulates basal disease resistance through the OsOxi1-OsPtila phosphorylation cascade in rice. Plant Cell Physiol. 51:2082-2091.

Matsui, H., Yamazaki, M., Kishi-Kaboshi, M., Takahashi, A., and Hirochika, H. 2010b. AGC kinase OsOxil positively regulates basal resistance through suppression of OsPtila-mediated negative regulation. Plant Cell Physiol. 51:1731-1744.

Meng, X., and Zhang, S. 2013. MAPK cascades in plant disease resistance signaling. Annu. Rev. Phytopathol. 51:245-266.

Mucyn, T. S., Clemente, A., Andriotis, V. M., Balmuth, A. L., Oldroyd, G. E., Staskawicz, B. J., and Rathjen, J. P. 2006. The tomato NBARCLRR protein Prf interacts with Pto kinase in vivo to regulate specific plant immunity. Plant Cell 18:2792-2806.

Nakagawa, T., Suzuki, T., Murata, S., Nakamura, S., Hino, T., Maeo, K., Tabata, R., Kawai, T., Tanaka, K., Niwa, Y., Watanabe, Y., Nakamura, K., Kimura, T., and Ishiguro, S. 2007. Improved Gateway binary vectors: High-performance vectors for creation of fusion constructs in transgenic analysis of plants. Biosci. Biotechnol. Biochem. 71:2095-2100.

Nguyen, H. P., Chakravarthy, S., Velásquez, A. C., McLane, H. L., Zeng, L., Nakayashiki, H., Park, D. H., Collmer, A., and Martin, G. B. 2010b. Methods to study PAMP-triggered immunity using tomato and Nicotiana benthamiana. Mol. Plant-Microbe Interact 23:991-999.

Nguyen, H. P., Yeam, I., Angot, A., and Martin, G. B. 2010a. Two virulence determinants of type III effector AvrPto are functionally conserved in diverse Pseudomonas syringae pathovars. New Phytol. 187:969-982.

Oh, C. S., Pedley, K. F., and Martin, G. B. 2010. Tomato 14-3-3 protein 7 positively regulates immunity-associated programmed cell death by enhancing protein abundance and signaling ability of MAPKKK $\alpha$. Plant Cell 22:260-272.

Pascuzzi, P. E. 2006. Structure-based functional analyses of Pseudomonas type III effector protein AvrPto and evaulation of putative virulence targets in tomato. Ph.D. thesis. Cornell University, Ithaca, NY, U.S.A.

Pedley, K. F., and Martin, G. B. 2003. Molecular basis of Pto-mediated resistance to bacterial speck disease in tomato. Annu. Rev. Phytopathol. 41:215-243

Pombo, M. A., Zheng, Y., Fernandez-Pozo, N., Dunham, D. M., Fei, Z., and Martin, G. B. 2014. Transcriptomic analysis reveals tomato genes whose expression is induced specifically during effector-triggered immunity and identifies the Epk1 protein kinase which is required for the host response to three bacterial effector proteins. Genome Biol. 15: 492.

Richau, K. H., Kaschani, F., Verdoes, M., Pansuriya, T. C., Niessen, S., Stüber, K., Colby, T., Overkleeft, H. S., Bogyo, M., and Van der Hoorn, R. A. 2012. Subclassification and biochemical analysis of plant papainlike cysteine proteases displays subfamily-specific characteristics. Plant Physiol. 158:1583-1599.

Robatzek, S., Bittel, P., Chinchilla, D., Köchner, P., Felix, G., Shiu, S. H., and Boller, T. 2007. Molecular identification and characterization of the tomato flagellin receptor LeFLS2, an orthologue of Arabidopsis FLS2 exhibiting characteristically different perception specificities. Plant Mol. Biol. 64:539-547.

Rosli, H. G., Zheng, Y., Pombo, M. A., Zhong, S., Bombarely, A., Fei, Z., Collmer, A., and Martin, G. B. 2013. Transcriptomics-based screen for genes induced by flagellin and repressed by pathogen effectors identifies a cell wall-associated kinase involved in plant immunity. Genome Biol. 14:R139.

Salmeron, J. M., Barker, S. J., Carland, F. M., Mehta, A. Y., and Staskawicz, B. J. 1994. Tomato mutants altered in bacterial disease resistance provide evidence for a new locus controlling pathogen recognition. Plant Cell 6: 511-520.

Salmeron, J. M., Oldroyd, G. E., Rommens, C. M., Scofield, S. R., Kim, H. S., Lavelle, D. T., Dahlbeck, D., and Staskawicz, B. J. 1996. Tomato $\operatorname{Prf}$ is a member of the leucine-rich repeat class of plant disease resistance genes and lies embedded within the Pto kinase gene cluster. Cell 86:123-133.
Scofield, S. R., Tobias, C. M., Rathjen, J. P., Chang, J. H., Lavelle, D. T., Michelmore, R. W., and Staskawicz, B. J. 1996. Molecular basis of genefor-gene specificity in bacterial speck disease of tomato. Science 274: 2063-2065

Segonzac, C., Feike, D., Gimenez-Ibanez, S., Hann, D. R., Zipfel, C., and Rathjen, J. P. 2011. Hierarchy and roles of pathogen-associated molecular pattern-induced responses in Nicotiana benthamiana. Plant Physiol. 156:687-699.

Senthil-Kumar, M., and Mysore, K. S. 2011. Caveat of RNAi in plants: The off-target effect. Methods Mol. Biol. 744:13-25.

Sessa, G., D’Ascenzo, M., Loh, Y. T., and Martin, G. B. 1998. Biochemical properties of two protein kinases involved in disease resistance signaling in tomato. J. Biol. Chem. 273:15860-15865.

Sessa, G., D'Ascenzo, M., and Martin, G.B. 2000. The major site of the Pti1 kinase phosphorylated by the Pto kinase is located in the activation domain and is required for Pto-Pti1 physical interaction. FEBS J. 267: 171-178.

Shan, L., He, P., Li, J., Heese, A., Peck, S. C., Nürnberger, T., Martin, G. B., and Sheen, J. 2008. Bacterial effectors target the common signaling partner BAK1 to disrupt multiple MAMP receptor-signaling complexes and impede plant immunity. Cell Host Microbe 4:17-27.

Shan, L., Thara, V. K., Martin, G. B., Zhou, J. M., and Tang, X. 2000. The Pseudomonas AvrPto protein is differentially recognized by tomato and tobacco and is localized to the plant plasma membrane. Plant Cell 12: 2323-2338

Shindo, T., Kaschani, F., Yang, F., Kovács, J., Tian, F., Kourelis, J., Hong, T. N., Colby, T., Shabab, M., Chawla, R., Kumari, S., Ilyas, M., Hörger, A. C., Alfano, J. R., and van der Hoorn, R. A. 2016. Screen of nonannotated small secreted proteins of Pseudomonas syringae reveals a virulence factor that inhibits tomato immune proteases. PLoS Pathog. 12:e1005874.

Shirasu, K. 2009. The HSP90-SGT1 chaperone complex for NLR immune sensors. Annu. Rev. Plant Biol. 60:139-164.

Stegmann, M., Monaghan, J., Smakowska-Luzan, E., Rovenich, H., Lehner, A., Holton, N., Belkhadir, Y., and Zipfel, C. 2017. The receptor kinase FER is a RALF-regulated scaffold controlling plant immune signaling. Science 355:287-289.

Takahashi, A., Agrawal, G. K., Yamazaki, M., Onosato, K., Miyao, A., Kawasaki, T., Shimamoto, K., and Hirochika, H. 2007. Rice Ptila negatively regulates $R A R 1$-dependent defense responses. Plant Cell 19: 2940-2951.

Tang, X., Frederick, R. D., Zhou, J., Halterman, D. A., Jia, Y., and Martin, G. B. 1996. Initiation of plant disease resistance by physical interaction of AvrPto and Pto kinase. Science 274:2060-2063.

van der Hoorn, R. A., and Kamoun, S. 2008. From guard to decoy: A new model for perception of plant pathogen effectors. Plant Cell 20: 2009-2017.

Wei, C. F., Kvitko, B. H., Shimizu, R., Crabill, E., Alfano, J. R., Lin, N. C., Martin, G. B., Huang, H. C., and Collmer, A. 2007. A Pseudomonas syringae pv. tomato DC3000 mutant lacking the type III effector HopQ11 is able to cause disease in the model plant Nicotiana benthamiana. Plant J. 51:32-46.

Wroblewski, T., Tomczak, A., and Michelmore, R. 2005. Optimization of Agrobacterium-mediated transient assays of gene expression in lettuce, tomato and Arabidopsis. Plant Biotechnol. J. 3:259-273.

Wu, A. J., Andriotis, V. M., Durrant, M. C., and Rathjen, J. P. 2004. A patch of surface-exposed residues mediates negative regulation of immune signaling by tomato Pto kinase. Plant Cell 16:2809-2821.

Wu, C. H., Belhaj, K., Bozkurt, T. O., Birk, M. S., and Kamoun, S. 2016. Helper NLR proteins NRC2a/b and NRC 3 but not NRC1 are required for Pto-mediated cell death and resistance in Nicotiana benthamiana. New Phytol. 209:1344-1352.

Xiang, T., Zong, N., Zou, Y., Wu, Y., Zhang, J., Xing, W., Li, Y., Tang, X., Zhu, L., Chai, J., and Zhou, J. M. 2008. Pseudomonas syringae effector AvrPto blocks innate immunity by targeting receptor kinases. Curr. Biol. 18:74-80.

Xiao, F., Lu, M., Li, J., Zhao, T., Yi, S. Y., Thara, V. K., Tang, X., and Zhou, J. M. 2003. Pto mutants differentially activate Prf-dependent, avrPtoindependent resistance and gene-for-gene resistance. Plant Physiol. 131: 1239-1249.

Xing, W., Zou, Y., Liu, Q., Liu, J., Luo, X., Huang, Q., Chen, S., Zhu, L., Bi, R., Hao, Q., Wu, J. W., Zhou, J. M., and Chai, J. 2007. The structural basis for activation of plant immunity by bacterial effector protein AvrPto. Nature 449:243-247.

Yamaguchi, K., Yamada, K., Ishikawa, K., Yoshimura, S., Hayashi, N., Uchihashi, K., Ishihama, N., Kishi-Kaboshi, M., Takahashi, A., Tsuge, S., Ochiai, H., Tada, Y., Shimamoto, K., Yoshioka, H., and Kawasaki, T. 2013. A receptor-like cytoplasmic kinase targeted by a plant pathogen 
effector is directly phosphorylated by the chitin receptor and mediates rice immunity. Cell Host Microbe 13:347-357.

Yeam, I., Nguyen, H. P., and Martin, G. B. 2010. Phosphorylation of the Pseudomonas syringae effector AvrPto is required for FLS2/BAK1independent virulence activity and recognition by tobacco. Plant J. 61: 16-24.

Young, J. M., Dye, D. W., and Wilkie, J. P. 1986. Bacterial speck. Pages 610-614 in: Vegetable Diseases and Their Control. A. F. Sherf, ed. J. Wiley and Sons, Inc, New York.

Zeng, W., Brutus, A., Kremer, J. M., Withers, J. C., Gao, X., Jones, A. D., and He, S. Y. 2011. A genetic screen reveals Arabidopsis stomatal and/or apoplastic defenses against Pseudomonas syringae pv. tomato DC3000. PLoS Pathog. 7:e1002291.

Zhou, J., Loh, Y. T., Bressan, R. A., and Martin, G. B. 1995. The tomato gene Ptil encodes a serine/threonine kinase that is phosphorylated by Pto and is involved in the hypersensitive response. Cell 83:925-935.

Zhou, J. M., and Chai, J. 2008. Plant pathogenic bacterial type III effectors subdue host responses. Curr. Opin. Microbiol. 11:179-185.
Zipfel, C. 2014. Plant pattern-recognition receptors. Trends Immunol. 35: 345-351.

Zipfel, C., and Oldroyd, G. E. 2017. Plant signalling in symbiosis and immunity. Nature 543:328-336.

\section{AUTHOR-RECOMMENDED INTERNET RESOURCES}

National Center for Biotechnology Information Sequence Read Archive: https://www.ncbi.nlm.nih.gov/sra

Nicotiana benthamiana Resources:

http://btiscience.org/our-research/enabling-technologies/nicotianabenthamiana

Pseudomonas-Plant Interaction database: http://www.pseudomonas-syringae.org

Sol Genomics database: https://solgenomics.net

Tomato Functional Genomics database: http://ted.bti.cornell.edu Tomato Functional Genomics Digital Expression (RNA-seq) Experiment List database: http://ted.bti.cornell.edu/cgi-bin/TFGD/digital/home.cgi 